\title{
Pointwise differentiability of higher order for sets
}

\author{
Ulrich Menne
}

April 17, 2018

\begin{abstract}
The present paper develops two concepts of pointwise differentiability of higher order for arbitrary subsets of Euclidean space defined by comparing their distance functions to those of smooth submanifolds. Results include that differentials are Borel functions, higher order rectifiability of the set of differentiability points, and a Rademacher result. One concept is characterised by a limit procedure involving inhomogeneously dilated sets.

The original motivation to formulate the concepts stems from studying the support of stationary integral varifolds. In particular, strong pointwise differentiability of every positive integer order is shown at almost all points of the intersection of the support with a given plane.
\end{abstract}

MSC-classes 2010 51M05 (Primary); 26B05, 49Q20 (Secondary).

Keywords Higher order pointwise differentiability $\cdot$ rectifiability $\cdot$ RademacherStepanov type theorem $\cdot$ stationary integral varifold.

\section{Introduction}

Suppose throughout the introduction that $k$ and $n$ are positive integers, $0 \leq \alpha \leq 1$, $\gamma=k$ if $\alpha=0$, and $\gamma=(k, \alpha)$ if $\alpha>0$. Before outlining the characterisations and the differentiability theory for pointwise differentiability of higher order for sets in Subsections 1.3 and 1.4, the notation is introduced in Subsection 1.1 and the simpler case of functions is reviewed in Subsection 1.2 The results on varifolds are summarised in Subsection 1.5

\subsection{Notation}

The notation is consistent with Federer [Fed69, pp. 669-671]. Therefore the term "class $\gamma$ " is used instead of the more common term "class $\mathscr{C}^{\gamma}$ ", see 2.4 and 2.10 .

Additionally, the following definitions are made. If $x \in \mathbf{R}^{n}$ and $A \subset \mathbf{R}^{n}$, then

$$
\operatorname{dist}(x, A)=\inf \{|x-a|: a \in A\}
$$

denotes the distance of $x$ to $A$. Suppose $m$ is an integer with $0 \leq m \leq n$. If $S$ is an $m$ dimensional subspace of $\mathbf{R}^{n}$, then $S_{\natural}$ denotes the orthogonal projection of $\mathbf{R}^{n}$ onto $S$ with $S_{\natural} \mid S=\mathbf{1}_{S}$ and $S^{\perp}=\operatorname{ker} S_{\natural}$ denotes the orthogonal complement of $S$ in $\mathbf{R}^{n}$ 1 The Grassmann manifold $\mathbf{G}(n, m)$ of $m$ dimensional subspaces

\footnotetext{
${ }^{1}$ Whenever $A$ is a set $\mathbf{1}_{A}$ denotes the identity map of $A$, see [Fed69 p. 669].
} 
of $\mathbf{R}^{n}$ is topologised by its injection into $\operatorname{Hom}\left(\mathbf{R}^{n}, \mathbf{R}^{n}\right)$ which maps $S \in \mathbf{G}(n, m)$ onto $S_{\natural}{ }^{2} \quad$ Moreover, the image of a set $A$ under a relation $f$ is denoted by

$$
f[A]=\{y:(x, y) \in f \text { for some } x \in A\},
$$

see Kelley [Kel75, p. 8]. In this regard all relations, in particular functions, are considered as subsets of $\operatorname{dmn} f \times \operatorname{im} f$, the product of their domain and image.

Finally, concerning varifolds, the notation is consistent with Allard All72.

\subsection{Higher order differentiability theory for functions}

Definition (classical, see 2.6 and 2.7). Suppose $m$ is an integer, $0<m<n$, $f: \mathbf{R}^{m} \rightarrow \mathbf{R}^{n-m}$, and $a \in \overline{\mathbf{R}}^{m}$.

Then $f$ is termed pointwise differentiable of order $\gamma$ at $a$ if and only if there exists a polynomial function $P: \mathbf{R}^{m} \rightarrow \mathbf{R}^{n-m}$ of degree at most $k$ such that ${ }^{3}$

$$
\begin{gathered}
\lim _{r \rightarrow 0+} r^{-k} \sup \{|f(x)-P(x)|: x \in \mathbf{B}(a, r)\}=0 \quad \text { if } \alpha=0, \\
\limsup _{r \rightarrow 0+} r^{-k-\alpha} \sup \{|f(x)-P(x)|: x \in \mathbf{B}(a, r)\}<\infty \quad \text { if } \alpha>0 .
\end{gathered}
$$

In this case $P$ is unique and the pointwise differentials of order $i$ of $f$ at a are defined by

$$
\text { pt } \mathrm{D}^{i} f(a)=\mathrm{D}^{i} P(a) \text { for } i=0, \ldots, k \text {. }
$$

These differentials are also called " $i$ th Peano derivatives", see Zibman Zib78.

The case $(k, \alpha)=(1,0)$ corresponds to classical differentiability. If $n-m=1$ and $f$ is convex, then $f$ is pointwise differentiable of order 2 at $\mathscr{L}^{m}$ almost all $a$ by Alexandrov's theorem, see for instance [EG15, Theorem 6.9]. Pointwise differentiability of order 2 also plays an important role in the study of viscosity solutions to nonlinear elliptic equations, see for instance Trudinger Tru89] and Caffarelli, Crandall, Kocan, and Świẹch CCKŚ96. Examples of arbitrary order of differentiability may be obtained from Rešetnjak's differentiability result for Sobolev functions, see Reš68a, $\}^{4}$ in conjunction with embedding theorems into continuous functions (see O'Neil ['N63] and Stein [Ste81] for related sharp results).

The present development of a higher order differentiability theory of sets aims at generalising the following theorem concerning functions. The latter is readily deduced from known results and included here for expository reasons.

Theorem A, see 4.6. Suppose $m$ is an integer, $0<m<n, f: \mathbf{R}^{m} \rightarrow \mathbf{R}^{n-m}$, and $X$ is the set of $a \in \mathbf{R}^{m}$ at which $f$ is pointwise differentiable of order $\gamma$ at $a$.

Then the following four statements hold.

(1) The functions $\mathrm{pt} \mathrm{D}^{i} f$ are Borel functions whose domains are Borel subsets of $\mathbf{R}^{m}$ for $i=0, \ldots, k$ and $X$ is a Borel subset of $\mathbf{R}^{m}$.

(2) There exists a sequence of functions $g_{j}: \mathbf{R}^{m} \rightarrow \mathbf{R}^{n-m}$ of class $\gamma$ such that $\left.\mathscr{L}^{m}\left(X \sim \bigcup_{j=1}^{\infty}\left\{x: f(x)=g_{j}(x)\right\}\right)=0\right]^{5}$

\footnotetext{
${ }^{2}$ Equivalently, the topology on $\mathbf{G}(n, m)$ is characterised by the requirement that $\mathbf{G}(n, m)$ becomes a homogeneous space through the canonical transitive left action of the orthogonal group $\mathbf{O}(n)$ on $\mathbf{G}(n, m)$, see [Fed69, 2.7.1 3.2.28(2) (4)].

${ }^{3}$ The symbol $\mathbf{B}(a, r)$ denotes the closed ball with centre $a$ and radius $r$, see [Fed69 2.8.1].

${ }^{4}$ The Russian original is Reš68b.

${ }^{5}$ The symbol $\mathscr{L}^{m}$ denotes the $m$ dimensional Lebesgue measure, see [Fed69, 2.6.5].
} 
(3) If $g: \mathbf{R}^{m} \rightarrow \mathbf{R}^{n-m}$ is of class $\gamma$ and $Y=\{y: f(y)=g(y)\}$, then

$$
\begin{gathered}
\operatorname{pt} \mathrm{D}^{i} f(a)=\mathrm{D}^{i} g(a) \quad \text { for } i=0, \ldots, k, \\
\lim _{r \rightarrow 0+} r^{-k-\alpha} \sup \{|f(x)-g(x)|: x \in \mathbf{B}(a, r)\}=0
\end{gathered}
$$

at $\mathscr{L}^{m}$ almost all $a \in X \cap Y$.

(4) If $\alpha=1$, then $f$ is pointwise differentiable of order $k+1$ at $\mathscr{L}^{m}$ almost all $a \in X$.

For differentiability in Lebesgue spaces $\mathbf{L}_{p}\left(\mathscr{L}^{m}, \mathbf{R}^{n-m}\right)$ with $1<p \leq \infty$ similar results to Theorem A were developed by Calderón and Zygmund, see CZ61, Theorems 5, 9, 10, 13]. The present proof of Theorem A mainly relies on a characterisation of almost everywhere approximate differentiability of order $\gamma$, see Isakov Isa87a $\left.\right|^{6}$ and some techniques from [Fed69]. A more detailed description of its proof will be given jointly with that of Theorem $\mathrm{B}$ for sets below.

\subsection{Defining and characterising higher order pointwise dif- ferentiability for sets}

For sets the first concept of pointwise differentiability is defined as follows.

Definition (see 3.3, 3.4 3.10, and 3.11). Suppose $A \subset \mathbf{R}^{n}$ and $a \in \mathbf{R}^{n}$.

Then $A$ is termed strongly pointwise differentiable of order $\gamma$ at $a$ if and only if there exist an integer $m$ with $0 \leq m \leq n, S \in \mathbf{G}(n, m)$, and a polynomial function $P: S \rightarrow S^{\perp}$ of degree at most $k$ such that $B=\{\chi+P(\chi): \chi \in S\}$ satisfies $a \in B$ and

$$
\begin{gathered}
\lim _{r \rightarrow 0+} r^{-k} \sup \{|\operatorname{dist}(x, A)-\operatorname{dist}(x, B)|: x \in \mathbf{B}(a, r)\}=0 \quad \text { if } \alpha=0, \\
\limsup _{r \rightarrow 0+} r^{-k-\alpha} \sup \{|\operatorname{dist}(x, A)-\operatorname{dist}(x, B)|: x \in \mathbf{B}(a, r)\}<\infty \quad \text { if } \alpha>0 .
\end{gathered}
$$

In this case $a$ belongs to the closure of $A, \operatorname{Tan}(A, a)=\operatorname{Tan}(B, a) 7^{7} m$ is determined by $A$ and $a$, a plane $S \in \mathbf{G}(n, m)$ is admissible in the definition if and only if $S^{\perp} \cap \operatorname{Tan}(A, a)=\{0\}$, and $P$ is determined by $k, A$, and $(a, S)$, see 3.4, 3.10, and 3.11. Unlike in the case of functions, $m$ may depend on $a$, and $S_{\natural}(a)$ need not to belong to the interior of $S_{\sharp}[A]$ relative to $S$.

All these remarks also hold with respect to the following weaker differentiability requirement which treats the sets $A$ and $B$ in an asymmetric manner.

Definition (see 3.3 and 3.12). Suppose $A \subset \mathbf{R}^{n}$ and $a \in \mathbf{R}^{n}$.

Then $A$ is termed pointwise differentiable of order $\gamma$ at $a$ if and only if there exist an integer $m$ with $0 \leq m \leq n, S \in \mathbf{G}(n, m)$, and a polynomial function $P: S \rightarrow S^{\perp}$ of degree at most $k$ such that $B=\{\chi+P(\chi): \chi \in S\}$ satisfies

\footnotetext{
${ }^{6}$ The Russian original is Isa87b].

${ }^{7}$ The tangent cone $\operatorname{Tan}(A, a)$ consists of all $v \in \mathbf{R}^{n}$ such that for $\varepsilon>0$ there exist $x \in A$ and $0<r<\infty$ such that $|x-a|<\varepsilon$ and $|r(x-a)-v|<\varepsilon$, see [Fed69 3.1.21]. In set-valued analysis this cone is called "contingent cone" of $A$ at $a$, see AF09 4.1.1].
} 
$a \in B$ and

$$
\begin{gathered}
\lim _{r \rightarrow 0+} r^{-1} \sup \{|\operatorname{dist}(x, A)-\operatorname{dist}(x, B)|: x \in \mathbf{B}(a, r)\}=0, \\
\lim _{r \rightarrow 0+} r^{-k} \sup \{\operatorname{dist}(x, B): x \in A \cap \mathbf{B}(a, r)\}=0 \quad \text { if } \alpha=0, \\
\limsup _{r \rightarrow 0+} r^{-k-\alpha} \sup \{\operatorname{dist}(x, B): x \in A \cap \mathbf{B}(a, r)\}<\infty \quad \text { if } \alpha>0 .
\end{gathered}
$$

The pointwise differential $\mathrm{pt}^{k} A$ of order $k$ of $A$ is the function whose domain is the set of $(a, S)$ such that these conditions are satisfied for some $m$ and $P$ with $\alpha=0$ and whose value at such $(a, S)$ equals $\mathrm{D}^{k}\left(P \circ S_{\natural}\right)(a)$.

Requiring $S=\operatorname{Tan}(A, a)$, one could define an equivalent notion of pointwise differentials of higher order whose domains are subsets of $\mathbf{R}^{n}$, see 3.13 The present definition is chosen for notational effectiveness.

The motivation for the asymmetric definition is that in case $\alpha=0$ it may be characterised by an inductive procedure considering pointwise limits of the distance functions associated to inhomogeneously dilated sets, suitably subtracting homogeneous polynomial functions of smaller degree at each step, see 3.22 In particular, if $A$ is pointwise differentiable of order 1 at $a$ and $S=\operatorname{Tan}(A, a)$, then pointwise differentiability of order 2 of $A$ at $a$ is equivalent to the requirement that for some homogeneous polynomial function $Q: S \rightarrow S^{\perp}$ of degree 2 , the sets $A_{s}=\left\{s^{-1} S_{\natural}(\chi-a)+s^{-2} S_{\natural}^{\perp}(\chi-a): \chi \in A\right\}$ and $B=\{\chi+Q(\chi): \chi \in S\}$ satisfy

$$
\lim _{s \rightarrow 0+} \operatorname{dist}\left(x, A_{s}\right)=\operatorname{dist}(x, B) \quad \text { for } x \in \mathbf{R}^{n},
$$

see 3.23 for alternate formulations of the last condition. Therefore pointwise differentiability of order 2 corresponds to "twice differentiability" as defined by the author in [Men12b, p. 2253]. Similar inhomogeneous dilations of order 2 with weak convergence of the correspondingly restricted Hausdorff measures to "approximate tangent paraboloids" occur in Anzellotti and Serapioni [AS94, §3].

Basic examples of sets which are $\mathscr{H}^{m}$ almost everywhere strongly pointwise differentiable of order 2 are relative boundaries of $m+1$ dimensional convex subsets of $\mathbf{R}^{n}$, see 3.17. However, the main motivation of the author to study higher order differentiability theory of sets is given by sets for which no local graphical representation is available.

In order to characterise the preceding two concepts, one may assume

$$
\begin{aligned}
& A \subset\left\{\chi:\left|S_{\natural}^{\perp}(\chi-a)\right| \leq \kappa\left|S_{\natural}(\chi-a)\right|\right\} \\
& \quad \text { for some integer } m \text { with } 0 \leq m \leq n, S \in \mathbf{G}(n, m), \text { and } 0 \leq \kappa<\infty
\end{aligned}
$$

by 3.10 In this case both pointwise differentiability and strongly pointwise differentiability are characterised in terms of vertical closeness of order $\gamma$ of $A$ near $a$ to a polynomial function $P: S \rightarrow S^{\perp}$ of degree at most $k$ and the behaviour of the set $S_{\natural}[A]$ near $S_{\natural}(a)$, see 3.11 . The difference of the two concepts is solely given by the degree to which $S_{\natural}[A]$ is required to cover $A$ near $S_{\natural}(a)$. This characterisation provides the basic link between the differentiability theory of sets and that of functions on which the further development rests.

It would also appear natural to investigate whether the regularity conditions, pointwise and strong pointwise differentiability of higher order, can be characterised in terms of the behaviour of the various higher order tangent sets 
of set-valued analysis designed to capture higher order tangential behaviour of possibly irregular sets, see [AF09, §4.7].

\subsection{Higher order differentiability theory for sets}

The next theorem summarises the main results of the present paper on differentiability theory of sets. Their formulation is completely analogous to Theorem A but their proofs are more complex.

Theorem B, see 5.2, 5.5, and 5.7. Suppose $m$ is an integer, $0 \leq m \leq n$, $A \subset \mathbf{R}^{n}, X$ is the set of $a \in \mathbf{R}^{n}$ such that $A$ is pointwise [strongly pointwise] differentiable of order $\gamma$ at a with $\operatorname{dim} \operatorname{Tan}(A, a)=m, Y$ is set of $a \in \mathbf{R}^{n}$ such that $A$ is pointwise differentiable of order 1 at a with $\operatorname{dim} \operatorname{Tan}(A, a)=m$, and $\tau: Y \rightarrow \mathbf{G}(n, m)$ satisfies $\tau(a)=\operatorname{Tan}(A, a)$ for $a \in Y$.

Then the following four statements hold.

(1) The function $\tau$ is a Borel function and its domain $Y$ is a Borel set, the functions $\mathrm{pt}^{i} A$ are Borel functions whose domains are Borel sets for $i=1, \ldots, k$, and the set $X$ is a Borel set.

(2) There exists a countable collection of $m$ dimensional submanifolds of class $\gamma$ of $\mathbf{R}^{n}$ covering $\mathscr{H}^{m}$ almost all of $X 8^{8}$

(3) If $B$ is an $m$ dimensional submanifold of class $\gamma$ of $\mathbf{R}^{n}$, then $\mathscr{H}^{m}$ almost all $a \in B \cap X$ satisfy $\mathrm{pt}^{i} A(a, \cdot)=\operatorname{pt} \mathrm{D}^{i} B(a, \cdot)$ for $i=0, \ldots, k$ and

$$
\begin{gathered}
\lim _{r \rightarrow 0+} r^{-k-\alpha} \sup \{\operatorname{dist}(x, B): x \in A \cap \mathbf{B}(a, r)\}=0 . \\
{\left[\lim _{r \rightarrow 0+} r^{-k-\alpha} \sup \{|\operatorname{dist}(x, A)-\operatorname{dist}(x, B)|: x \in \mathbf{B}(a, r)\}=0 .\right]}
\end{gathered}
$$

(4) If $\alpha=1$, then $A$ is pointwise [strongly pointwise] differentiable of order $k+1$ at $\mathscr{H}^{m}$ almost all $a \in X$.

In order to prove $A(1)$ and $B(1)$, the main task is to prove that the functions in question are Borel subsets of suitable complete, separable metric spaces. By a classical result in descriptive set theory, see 4.1, this then readily yields (1). This pattern of proof is taken from [Fed69, 3.1.1]. As the natural domain of the polynomial functions associated with the differentials of sets, the tangent plane, depends on the point considered, some additional considerations, see 5.3 and 5.4, are needed to prove closedness of the auxiliary sets in the case of sets.

The proofs of $A(2)$ and $B(2)$ rely on (1) and a direct consequence, see 4.2 of Isakov's characterisation of functions which are almost everywhere approximately differentiable of order $\gamma$, see Isa87a. Whereas the case of functions is immediate, the case of sets requires the construction of an auxiliary function to which 4.2 can be applied. The function is constructed using the countable $m$ rectifiability ${ }^{9}$ of $X$, see 5.2 It inherits the higher order differentiability properties of $A$ by 5.6

The proofs of $\mathrm{A}(3)$ and $\mathrm{B}(3)$ rest on a special case of a differentiability theorem for functions on varifolds from Kolasiński and the author [KM17, 4.4].

\footnotetext{
${ }^{8}$ The symbol $\mathscr{H}^{m}$ denotes the $m$ dimensional Hausdorff measure, see Fed69, 2.10.2].

${ }^{9}$ A subset of $\mathbf{R}^{n}$ is called countably $m$ rectifiable if and only if it can be covered by the union of a countable family of Lipschitzian images of subsets of $\mathbf{R}^{m}$, see [Fed69. 3.2.14(2)].
} 
The short proof of the presently required case is included in 4.4 for the convenience of the reader. In the case of functions, one may directly apply 4.4 to the modulus of the difference of the two functions involved. In the case of sets, one instead applies that theorem to a sequence of auxiliary functions constructed so as to encode all necessary information on the relative position of the sets $A$ and $B$.

Finally, as in Liu Liu08, 1.6], A, (4) and B, (4) follow from the two statements respectively preceding it in conjunction with a Lusin type approximation of functions of class $(k, 1)$ by functions of class $k+1$, see [Fed69, 3.1.15].

\subsection{An application to stationary integral varifolds}

The following are two important questions for integral varifolds (a concept of generalised submanifolds with positive integer multiplicity) which either have "bounded mean curvature and no boundary" or are stationary.

Questions. Suppose $0 \leq \kappa<\infty, V$ is an at least two-dimensional integral varifold in $\mathbf{R}^{n},\|\delta V\| \leq \kappa\|V\|$, and $A=\operatorname{spt}\|V\|$.

(1) How regular needs $A$ to be near $\mathscr{H}^{m}$ almost all of its points?

(2) How much more regular needs $A$ to be if $\kappa=0$, i.e. if $V$ is stationary?

Calling a point of $A$ regular if and only if it possesses a neighbourhood $U$ such that $A \cap U$ is a submanifold of class 1 of $\mathbf{R}^{n}$, it is known that (1) does not entail regularity $\mathscr{H}^{m}$ almost everywhere; in fact, a sequence of increasingly irregular examples was constructed by Allard [All72, 8.1(2)], Brakke [Bra78 6.1], and Kolasiński and the author [KM17, 10.3, 10.8]. On the other hand various weaker properties almost everywhere resembling the behaviour of closed submanifolds of class 2 were established under the hypotheses of (1) by Brakke [Bra78, 5.8], Schätzle [Sch04, Theorems 4.1, 5.1, 6.1, 6.2] and [Sch09, Theorems 3.1, 4.1], White [Whi10, Theorem 2], and the author [Men10, 4.11], Men12a 10.2], [Men13, 4.8, 5.2], [Men16a, 14.2], and [Men16b, 6.8].

The only regularity properties valid across points with higher multiplicity and specific to the stationary case, see (2), are a number of increasingly delicate maximum principles by Solomon and White [SW89, Ilmanen [Ilm96, Theorem A], and Wickramasekera [Wic14a, Theorem 19.1] and [Wic14b, Theorem 1.1]. The present paper makes the first contribution to (2) valid across points with higher multiplicity which is different from a maximum principle.

Theorem C, see 7.3. Suppose $m$ is an integer, $2 \leq m \leq n, S \in \mathbf{G}(n, m), V$ is an $m$ dimensional stationary integral varifold in $\mathbf{R}^{n}$, and $A=\operatorname{spt}\|V\|$.

Then $A$ is strongly pointwise differentiable of every positive integer order at $\mathscr{H}^{m}$ almost all $a \in A \cap S$.

In 6.7 an example is constructed that shows that Theorem C constitutes a regularity property of integral varifolds setting the stationary case apart of that of bounded mean curvature and no boundary, see 7.4 As far as pointwise differentiability is concerned, it relies on "local maximum estimates" for subsolutions to the Laplace equation on varifolds by Michael and Simon MS73, 3.4] and the general principle that control in an approximate sense tends to entail control in an integral sense in the presence of an elliptic partial differential equation. This principle was first discovered by Schätzle in [Sch09, Theorem 3.1] and was since used by author in [Men13, 5.2] and jointly with Kolasiński in [KM17. 
9.2]. Its present implementation in 7.1 using Michael and Simon's result is considerably simpler than previous approaches. Strong pointwise differentiability then follows using a result of Kolasiński and the author [KM17, 10.4] which relies on Almgren's multiple valued functions from [Alm00.

Theorem C naturally raises the following regularity question for stationary integral varifolds that appears to be more tractable than that of possible almost everywhere regularity: Suppose $V$ is an $m$ dimensional stationary integral varifold in $\mathbf{R}^{n}$ and $A=\operatorname{spt}\|V\|$. Is A necessarily [strongly] pointwise differentiable of every positive integer order at $\mathscr{H}^{m}$ almost all $a \in A$ ?

It has been announced by the author in Men12b, Corollary 2(1)] that under weaker hypotheses than those of (1) the set $A$ is pointwise differentiable of order 2 at $\mathscr{H}^{m}$ almost all of its points. Theorem $\mathrm{B}$ is a significantly generalised version of the first part of the proof of that result. The second part of the proof along with some generalisations of Theorem C shall appear elsewhere. The formulation of Theorem $\mathrm{B}$ as a separate result for general subsets of Euclidean space shall facilitate the use of this technique outside the varifold context.

\subsection{Subsequent developments}

Since the publication of the initial version of this paper, two sequels have been written. Firstly, Santilli introduced the corresponding approximate notion of pointwise differentiability for sets (see [San17, 3.8, 3.19] and the footnote to 3.12). He thus obtains a characterisation of higher order rectifiability, see [San17, 1.2]; a problem left open by Anzellotti and Serapioni in AS94. Secondly, the author developed an analogous theory of pointwise differentiability of higher order for distributions in Men18. The latter is related to the afore-mentioned regularity question for stationary integral varifolds as is elaborated upon in [Men18, §1.3].

\subsection{Organisation of the paper}

After Section 2 on preliminaries, Section 3 provides definitions and characterisations for higher order differentiability of sets. Sections 4 and 5 treat the higher order differentiability theory for functions and sets respectively. In Section 6 an example for use in Section 7 on varifolds is constructed. Finally, Appendix A contains a table with brief descriptions of the items employed from [Fed69].

\subsection{Acknowledgements}

The author would like to thank Mario Santilli for reading part of the manuscript and for bringing a series of papers of Isakov to his attention, Dr Sławomir Kolasiński for helping him to become acquainted with some of these results available only in Russian, and Dr Yangqin Fang for pointing him to [Dav03]. The initial version of this paper (see $\operatorname{arXiv:1603.08587v1)}$ was written while the author worked at the Max Planck Institute for Gravitational Physics (Albert Einstein Institute) and the University of Potsdam.

\section{Preliminaries}

In this section, firstly, an a priori estimate for polynomial functions, see 2.1. and a resulting uniqueness theorem, see 2.3 are proven. Secondly, classical notions 
of higher order differentiability are compiled, see 2.4 2.7, and 2.10.

2.1 Lemma. Suppose $1 \leq M<\infty$.

Then there exists a positive, finite number $\Gamma$ with the following property.

If $k$ is a nonnegative integer, $k \leq M, S$ is a Hilbert space, $\operatorname{dim} S \leq M, a \in S$, $0<r<\infty, Y$ is a normed vectorspace, $P: S \rightarrow Y$ is a polynomial function of degree at most $k$, and $X \subset \mathbf{B}(a, r)$ satisfies $\operatorname{dist}(x, X) \leq \Gamma^{-1} r$ for $x \in \mathbf{B}(a, r)$, then there holds

$$
\sup \left\{r^{i}\left\|\mathrm{D}^{i} P(x)\right\|: x \in \mathbf{B}(a, r), i=0, \ldots, k\right\} \leq \Gamma \sup \{|P(x)|: x \in X\} .
$$

Proof. Using translations, homotheties, and the equation

$$
|y|=\sup \left\{|\alpha(y)|: \alpha \in Y^{*} \text { and }\|\alpha\| \leq 1\right\} \quad \text { for } y \in Y,
$$

see [DS58, II.3.15], it is sufficient to prove the assertion resulting from additionally hypothesising $a=0, r=1$, and $Y=\mathbf{R}$ in the body of the lemma.

If the remaining assertion were false for some $M$, there would exist a sequence $\Gamma_{j}$ with $\Gamma_{j} \rightarrow \infty$ as $j \rightarrow \infty$ and sequences $k_{j}, S_{j}, P_{j}$, and $X_{j}$ showing that $\Gamma_{j}$ does not have the property described in the remaining assertion. One could assume that, for some $k$ and $S$, there would hold

$$
k_{j}=k, \quad S_{j}=S, \quad \sup \left\{\left\|\mathrm{D}^{i} P_{j}(x)\right\|: x \in S \cap \mathbf{B}(0,1), i=0, \ldots, k\right\}=1
$$

for every positive integer $j$. Since the space of polynomial functions $P: S \rightarrow \mathbf{R}$ of degree at most $k$ is finite dimensional and the function on that space with value

$$
\sup \left\{\left\|\mathrm{D}^{i} P(x)\right\|: x \in S \cap \mathbf{B}(0,1), i=0, \ldots, k\right\}
$$

at $P$ is a norm, see [Fed69, 1.10.2 1.10.4 3.1.11, possibly passing to a subsequence, there would exist a polynomial function $P: S \rightarrow \mathbf{R}$ such that

$$
\begin{gathered}
\sup \left\{\left\|\mathrm{D}^{i} P(x)\right\|: x \in S \cap \mathbf{B}(0,1), i=0, \ldots, k\right\}=1, \\
\sup \{|P(x)|: x \in S \cap \mathbf{B}(0,1)\} \leq \liminf _{j \rightarrow \infty} \sup \left\{\left|P_{j}(x)\right|: x \in X_{j}\right\}=0 .
\end{gathered}
$$

This would be a contradiction.

2.2 Remark. A conceptually similar lemma appears in Campanato Cam64, Lemma 2.I] and is attributed there to De Giorgi.

2.3 Theorem. Suppose $k$ is a nonnegative integer, $S$ is a Hilbert space, $\operatorname{dim} S<$ $\infty, Y$ is a normed vectorspace, $P: S \rightarrow Y$ is a polynomial function of degree at most $k, a \in S, X \subset S$, and

$$
\lim _{r \rightarrow 0+} r^{-1} \sup \operatorname{dist}(\cdot, X)[S \cap \mathbf{B}(a, r)]=0, \quad \lim _{r \rightarrow 0+} r^{-k} \sup |P|[X \cap \mathbf{B}(a, r)]=0 .
$$

Then $P=0$.

Proof. Applying 2.1 for each sufficiently small $r$ yields $\mathrm{D}^{i} P(a)=0$ for $i=$ $0, \ldots, k$, hence $P=0$ by [Fed69, 3.1.11. 
2.4 Definition. Suppose $k$ is a nonnegative integer, $0<\alpha \leq 1, X$ and $Y$ are normed vectorspaces, and $f$ maps a subset of $X$ into $Y$.

Then $f$ is said to be of class $(k, \alpha)$ if and only if $f$ is of class $k$ and $\mathrm{D}^{k} f$ locally satisfies a Hölder condition with exponent $\alpha 10$ Moreover, $f$ is called a diffeomorphism of class $(k, \alpha)$ if and only if $f$ is a homeomorphism, $f$ is of class $(k, \alpha)$, and $f^{-1}$ is of class $(k, \alpha)$.

2.5. Suppose $k$ is a nonnegative integer, $0<\alpha \leq 1, m$ is a positive integer, $U$ is an open subset of $\mathbf{R}^{m}, Y$ is a normed vectorspace, and $f_{i}: U \rightarrow \bigodot^{i}\left(\mathbf{R}^{m}, Y\right)$ for $i=0, \ldots, k{ }^{11}$ Then [Fed69, 3.1.11 3.1.14 and 2.1 may be used to verify that $f_{0}$ is of class $(k, \alpha)$ with $\mathrm{D}^{i} f_{0}=f_{i}$ for $i=0, \ldots, k$ if and only if

$$
\sup \left\{|x-a|^{-k-\alpha}\left|f_{0}(x)-\sum_{i=0}^{k}\left\langle(x-a)^{i} / i !, f_{i}(a)\right\rangle\right|: a, x \in K, a \neq x\right\}<\infty
$$

whenever $K$ is a compact subset of $U \mathbb{1 2}$

2.6. Suppose $k$ is a nonnegative integer, $0 \leq \alpha \leq 1, \gamma=k$ if $\alpha=0$ and $\gamma=(k, \alpha)$ if $\alpha>0, X$ and $Y$ are normed vectorspaces, $a \in U \subset X, f: U \rightarrow Y$ is of class $\gamma$, and $P$ is the $k$ jet of $f$ at $a !^{13}$ Then [Fed69, 3.1.11] yields

$$
\begin{gathered}
\lim _{x \rightarrow a}|f(x)-P(x)| /|x-a|^{k}=0 \quad \text { if } \alpha=0, \\
\limsup _{x \rightarrow a}|f(x)-P(x)| /|x-a|^{k+\alpha}<\infty \quad \text { if } \alpha>0 .
\end{gathered}
$$

2.7 Definition. Suppose $k$ is a nonnegative integer, $0 \leq \alpha \leq 1, \gamma=k$ if $\alpha=0$ and $\gamma=(k, \alpha)$ if $\alpha>0, X$ and $Y$ are normed vectorspaces, $f$ maps a subset of $X$ into $Y$, and $a \in X$.

Then $f$ is called pointwise differentiable of order $\gamma$ at $a$ if and only if there exist an open subset $U$ of $X$ and a function $g: U \rightarrow Y$ of class $\gamma$ such that

$$
\begin{gathered}
a \in U \subset \operatorname{dmn} f, \quad f(a)=g(a), \\
\lim _{x \rightarrow a}|f(x)-g(x)| /|x-a|^{k}=0 \quad \text { if } \alpha=0, \\
\limsup _{x \rightarrow a}|f(x)-g(x)| /|x-a|^{k+\alpha}<\infty \quad \text { if } \alpha>0 .
\end{gathered}
$$

Whenever $f$ is pointwise differentiable of order $k$ at $a$ one defines (see 2.6) the pointwise differential of order $i$ of $f$ at a by

$$
\operatorname{pt} \mathrm{D}^{i} f(a)=\mathrm{D}^{i} g(a) \text { for } i=0, \ldots, k .
$$

\footnotetext{
${ }^{10}$ The map $f$ is called of class $k$ if and only if its domain is open and it is $k$ times continuously differentiable, see [Fed69] 3.1.11].

${ }^{11}$ If $V$ and $W$ are vectorspaces, then $\bigodot^{0}(V, W)=W$ and $\bigodot^{i}(V, W)$ is the vectorspace of all symmetric $i$ linear maps from $V^{i}$ into $W$ whenever $i$ is a positive integer, see Fed69, 1.10.1].

${ }^{12}$ If $V$ and $W$ are vectorspaces, $i$ is a positive integer, and $\phi \in \bigodot^{i}(V, W)$, then$$
\left\langle v^{i} / i !, \phi\right\rangle=i !^{-1} \phi(v, \ldots, v) \quad \text { for } v \in V,
$$

see [Fed69, 1.9.1, 1.10.1, 1.10.4]. Similarly, $\left\langle v^{i} / i !, \phi\right\rangle=\phi$ if $i=0$ and $\phi \in \bigodot^{0}(V, W)$.

${ }^{13}$ The $k$ jet of $f$ at $a$ is the polynomial function $P: X \rightarrow Y$ of degree at most $k$ satisfying $P(x)=\sum_{i=0}^{k}\left\langle(x-a)^{i} / i\right.$ ! , $\left.\mathrm{D}^{i} f(a)\right\rangle$ for $x \in X$, see Fed69 3.1.11].
} 
2.8 Remark. A function is pointwise differentiable of order 0 at $a$ if and only

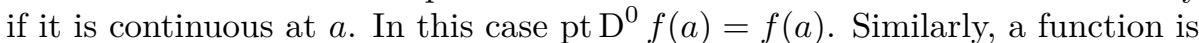
pointwise differentiable of order 1 at $a$ if and only if it is differentiable at $a$ in which case pt $\mathrm{D}^{1} f(a)=\mathrm{D} f(a)$.

2.9 Remark. If $f$ is $k$ times differentiable at $a$, then $f$ is pointwise differentiable of order $k$ at $a$ and $\operatorname{ptD}^{i} f(a)=\mathrm{D}^{i} f(a)$ for $i=0, \ldots, k$; in fact, one may employ an induction argument based on the fact that $\operatorname{Lip} f=\sup \|\mathrm{D} f\|[U]$ whenever $U$ is open and convex with $U \subset \operatorname{dmn} \mathrm{D} f$, see Fed69, 2.2.7, 3.1.1, the one-dimensional case on which appears in Weil [Wei95, p. 589] ${ }^{14}$

2.10 Definition. Suppose $k$ and $n$ are positive integers, $m$ is an integer, $0 \leq$ $m \leq n, 0<\alpha \leq 1$, and $B \subset \mathbf{R}^{n}$.

Then $B$ is called an $m$ dimensional submanifold of class $(k, \alpha)$ if and only if for each $b \in B$ there exists a neighbourhood $U$ of $b$ in $\mathbf{R}^{n}$, a diffeomorphism $f: U \rightarrow \mathbf{R}^{n}$ of class $(k, \alpha)$, and an $m$ dimensional subspace $T$ of $\mathbf{R}^{n}$ with

$$
f[B \cap U]=T \cap \operatorname{im} f .
$$

2.11 Remark. The basic properties of maps and submanifolds of class $k$ given in Fed69, 3.1.18, 3.1.19 remain valid for maps and submanifolds of class $(k, \alpha)$.

\section{Basic characterisations}

In the present section the key definitions concerning differentiability of higher order for sets are provided in 3.3 and 3.12 . The main characterisations of these concepts are proven in 3.11 and 3.22 the first of which takes a particularly simple form if the set is associated to the graph of a function, see 3.14

3.1. Suppose $A \subset \mathbf{R}^{n}$ and $a \in \mathbf{R}^{n}$. Then $\operatorname{Tan}(A, a)=\{0\}$ if and only if $a$ is an isolated point of $A$, as may be verified using [Fed69, 3.1.21.

3.2. Suppose $A \subset \mathbf{R}^{n}, B \subset \mathbf{R}^{n}, a \in(\operatorname{Clos} A) \cap(\operatorname{Clos} B)$, and $0<r<\infty .15$ Then one verifies 16

$$
\begin{aligned}
& \sup \mid \operatorname{dist}(\cdot, A)-\operatorname{dist}(\cdot, B) \mid[\mathbf{U}(a, r)] \\
& \leq \sup (\operatorname{dist}(\cdot, A)[B \cap \mathbf{U}(a, 2 r)] \cup \operatorname{dist}(\cdot, B)[A \cap \mathbf{U}(a, 2 r)]) .
\end{aligned}
$$

3.3 Definition. Suppose $k$ and $n$ are positive integers, $0 \leq \alpha \leq 1, \gamma=k$ if $\alpha=0$ and $\gamma=(k, \alpha)$ if $\alpha>0$, and $A \subset \mathbf{R}^{n}$.

Then $A$ is called pointwise [strongly pointwise] differentiable of order $\gamma$ at a if and only if there exists a submanifold $B$ of class $\gamma$ of $\mathbf{R}^{n}$ such that $a \in B$,

$$
\lim _{r \rightarrow 0+} r^{-1} \sup |\operatorname{dist}(\cdot, A)-\operatorname{dist}(\cdot, B)|[\mathbf{B}(a, r)]=0,
$$

\footnotetext{
${ }^{14}$ If $g$ is a map between metric spaces, then $\operatorname{Lip} g$ is its Lipschitz constant, see [Fed69, 2.2.7].

${ }^{15}$ The closure of a set $A$ is denoted Clos $A$, see [Fed69, p. 669].

${ }^{16}$ The symbol $\mathbf{U}(a, r)$ denotes the open ball with centre $a$ and radius $r$, see [Fed69.8.1.
} 
and

$$
\begin{gathered}
\lim _{r \rightarrow 0+} r^{-k} \sup \operatorname{dist}(\cdot, B)[A \cap \mathbf{B}(a, r)]=0 \quad \text { if } \alpha=0, \\
\limsup _{r \rightarrow 0+} r^{-k-\alpha} \sup \operatorname{dist}(\cdot, B)[A \cap \mathbf{B}(a, r)]<\infty \quad \text { if } \alpha>0 . \\
{\left[\begin{array}{c}
\lim _{r \rightarrow 0+} r^{-k} \sup |\operatorname{dist}(\cdot, A)-\operatorname{dist}(\cdot, B)|[\mathbf{B}(a, r)]=0 \quad \text { if } \alpha=0, \\
\limsup _{r \rightarrow 0+} r^{-k-\alpha} \sup |\operatorname{dist}(\cdot, A)-\operatorname{dist}(\cdot, B)|[\mathbf{B}(a, r)]<\infty \quad \text { if } \alpha>0 .
\end{array}\right]}
\end{gathered}
$$

3.4 Remark. It follows that $a \in \operatorname{Clos} A$. Moreover, one verifies $\operatorname{Tan}(A, a)=$ $\operatorname{Tan}(B, a)$, in particular $\operatorname{Tan}(A, a)$ is a $\operatorname{dim} B$ dimensional subspace of $\mathbf{R}^{n}$.

3.5 Remark. In the bracketed case the condition

$$
\lim _{r \rightarrow 0+} r^{-1} \sup |\operatorname{dist}(\cdot, A)-\operatorname{dist}(\cdot, B)|[\mathbf{B}(a, r)]=0
$$

is redundant.

3.6 Remark. If $(k, \alpha)=(1,0)$, then pointwise differentiability and strong pointwise differentiability agree.

3.7 Remark. If $U$ is an open subset of $\mathbf{R}^{n}, g: U \rightarrow \mathbf{R}^{n}$ is a diffeomorphism of class $\gamma, A \subset U, a \in U$, and $A$ is pointwise [strongly pointwise] differentiable of order $\gamma$ at $a$, then $g[A]$ is pointwise [strongly pointwise] differentiable of order $\gamma$ at $g(a)$, as may be verified using 3.2

3.8 Remark. Suppose $A, k, \alpha$, and $\gamma$ are as the hypotheses of 3.3 Then $A$ is pointwise [strongly pointwise] differentiable of order $\gamma$ at $a \in \mathbf{R}^{n}$ with $\operatorname{Tan}(A, a) \in \mathbf{G}(n, 0)$ if and only if $a$ is isolated in $A$ by 3.1

3.9 Example. Suppose $n$ is a positive integer, $m$ is an integer, $0 \leq m \leq n$, and $A \subset S \in \mathbf{G}(n, m)$. Then $A$ is pointwise differentiable of order 1 at $a$ with $\operatorname{Tan}(A, a)=S$ whenever $a \in S$ and $\Theta^{m}\left(\mathscr{H}^{m}\llcorner S \sim \operatorname{Clos} A, a)=0\right.$ which is the case at $\mathscr{H}^{m}$ almost all $a \in \operatorname{Clos} A$ by [Fed69, 2.10.19](4)] ${ }^{17}$

3.10. If $n$ is a positive integer, $m$ is an integer, $0 \leq m \leq n, A \subset \mathbf{R}^{n}, a \in \operatorname{Clos} A$, $S \in \mathbf{G}(n, m)$, and $S^{\perp} \cap \operatorname{Tan}(A, a)=\{0\}$, then there exist $r>0$ and $0 \leq \kappa<\infty$ such that

$$
A \cap \mathbf{B}(a, r) \subset\left\{\chi:\left|S_{\natural}^{\perp}(\chi-a)\right| \leq \kappa\left|S_{\natural}(\chi-a)\right|\right\} .
$$

3.11 Theorem. Suppose $k$ and $n$ are positive integers, $m$ is an integer, $0 \leq$ $m \leq n, 0 \leq \alpha \leq 1, \gamma=k$ if $\alpha=0$ and $\gamma=(k, \alpha)$ if $\alpha>0, A \subset \mathbf{R}^{n}, a \in \operatorname{Clos} A$, $S \in \mathbf{G}(n, m), 0 \leq \kappa<\infty$, and

$$
A \subset\left\{\chi:\left|S_{\natural}^{\perp}(\chi-a)\right| \leq \kappa\left|S_{\natural}(\chi-a)\right|\right\} .
$$

Then the following three conditions are equivalent.

(1) There exists an $m$ dimensional submanifold $B$ of class $\gamma$ of $\mathbf{R}^{n}$ such that $B$ satisfies the conditions of 3.3 .

\footnotetext{
${ }^{17}$ The $m$ dimensional density of a measure $\phi$ over $\mathbf{R}^{n}$ at $a$ equals$$
\Theta^{m}(\phi, a)=\lim _{r \rightarrow 0+} \frac{\phi \mathbf{B}(a, r)}{\boldsymbol{\alpha}(m) r^{m}}
$$

where $\boldsymbol{\alpha}(m)=\mathscr{L}^{m} \mathbf{B}(0,1)$ if $m>0$ and $\boldsymbol{\alpha}(0)=1$, see Fed69 2.7.16 (1), 2.10.19].
} 
(2) The set $S_{\natural}[A]$ is strongly pointwise differentiable of order 1 [order $\left.\gamma\right]$ at $S_{\natural}(a)$ with $\operatorname{Tan}\left(S_{\natural}[A], S_{\natural}(a)\right)=S$ and there exists an $m$ dimensional submanifold $B$ of class $\gamma$ of $\mathbf{R}^{n}$ such that $a \in B$ and

$$
\begin{gathered}
\lim _{r \rightarrow 0+} r^{-k} \sup \operatorname{dist}(\cdot, B)[A \cap \mathbf{B}(a, r)]=0 \quad \text { if } \alpha=0, \\
\limsup _{r \rightarrow 0+} r^{-k-\alpha} \sup \operatorname{dist}(\cdot, B)[A \cap \mathbf{B}(a, r)]<\infty \quad \text { if } \alpha>0 .
\end{gathered}
$$

(3) The set $S_{\natural}[A]$ is strongly pointwise differentiable of order 1 [order $\left.\gamma\right]$ at $S_{\natural}(a)$ with $\operatorname{Tan}\left(S_{\natural}[A], S_{\natural}(a)\right)=S$ and there exists a function $f: S \rightarrow S^{\perp}$ of class $\gamma$ satisfying

$$
\lim _{r \rightarrow 0+} r^{-k} \sup \left\{\left|S_{\natural}^{\perp}(\chi)-f\left(S_{\natural}(\chi)\right)\right|: \chi \in A \cap S_{\natural}^{-1}\left[\mathbf{B}\left(S_{\natural}(a), r\right)\right]\right\}=0
$$

if $\alpha=0$, and

$$
\limsup _{r \rightarrow 0+} r^{-k-\alpha} \sup \left\{\left|S_{\natural}^{\perp}(\chi)-f\left(S_{\natural}(\chi)\right)\right|: \chi \in A \cap S_{\natural}^{-1}\left[\mathbf{B}\left(S_{\natural}(a), r\right)\right]\right\}<\infty
$$

if $\alpha>0$.

In this case the $k$ jet $P$ of $f$ at $S_{\natural}(a)$ is uniquely determined by $k, A$, and $(a, S)$ and the following three additional statements hold.

(4) An $m$ dimensional submanifold $B$ of class $\gamma$ of $\mathbf{R}^{n}$ satisfies (1) if and only if there exists a function $f: S \rightarrow S^{\perp}$ of class $\gamma$ such that

$$
B \cap U=\{\chi+f(\chi): \chi \in S\} \cap U \quad \text { for some neighbourhood } U \text { of a }
$$

and the $k$ jet of $f$ at $S_{\natural}(a)$ equals $P$.

(5) The statement (4) holds with "(1)" replaced by "(22)".

(6) A function $f: S \rightarrow S^{\perp}$ of class $\gamma$ satisfies (3) if and only if its $k$ jet at $S_{\natural}(a)$ equals $P$.

Proof. If $m=0$ then $A=\{a\}$. Therefore one may assume $m>0$ by 3.8

Clearly, (3) implies (2) with $B=\{\chi+f(\chi): \chi \in S\}$. Moreover, if $B$ satisfies (1) then there exists a function $f: S \rightarrow S^{\perp}$ of class $\gamma$ such that

$$
B \cap U=\{\chi+f(\chi): \chi \in S\} \cap U \text { for some neighbourhood } U \text { of } a
$$

by 2.11, 3.4, and [Fed69, 3.1.18, 3.1.19 (4)].

Next, two implications will be shown.

Firstly, if $B$ satisfies (2) then $B$ satisfies (1). In this regard, notice that

$$
\operatorname{Tan}(A, a) \subset \operatorname{Tan}(B, a), \quad \operatorname{Tan}\left(S_{\natural}[A], S_{\natural}(a)\right) \subset S_{\natural}[\operatorname{Tan}(A, a)],
$$

where the second inclusion is implied by $S^{\perp} \cap \operatorname{Tan}(A, a)=\{0\}$. It follows $S_{\natural}[\operatorname{Tan}(B, a)]=S$, the linear map $S_{\natural} \mid \operatorname{Tan}(B, a)$ is univalent 18 and

$$
\operatorname{Tan}(A, a)=\operatorname{Tan}(B, a) .
$$

\footnotetext{
${ }^{18}$ The term "univalent" is also known as "injective".
} 
Therefore, in view of [Fed69, 3.1.18 3.1.19(4)], one may assume

$$
\left|S_{\natural}^{\perp}(\chi-b)\right| \leq \kappa\left|S_{\natural}(\chi-b)\right| \quad \text { whenever } \chi, b \in B
$$

possibly enlarging $\kappa$ and shrinking $B$. Also observe that 3.4 with $A, a$, and $B$ replaced by $S_{\natural}[A], S_{\natural}(a)$, and the submanifold furnished by the strong pointwise differentiability of order 1 [order $\gamma]$ of $S_{\natural}[A]$ at $S_{\natural}(a)$, Fed69, 3.1.19 (5)], and

$$
\operatorname{dist}\left(S_{\natural}(\chi), S_{\natural}[A]\right) \leq \operatorname{dist}\left(\chi, S_{\natural}[A]\right) \quad \text { for } \chi \in \mathbf{R}^{n}
$$

imply for $\delta(r)=\sup \operatorname{dist}\left(\cdot, S_{\natural}[A]\right)\left[S \cap \mathbf{B}\left(S_{\natural}(a), r\right)\right]$ that

$$
\begin{gathered}
\lim _{r \rightarrow 0+} r^{-1} \delta(r)=0 . \\
{\left[\lim _{r \rightarrow 0+} r^{-k} \delta(r)=0 \quad \text { if } \alpha=0, \quad \limsup _{r \rightarrow 0+} r^{-k-\alpha} \delta(r)<\infty \quad \text { if } \alpha>0 .\right.}
\end{gathered}
$$

If $\chi \in B$ and $|\chi-a| \leq r$, then, as $\operatorname{Clos} S_{\mathrm{\natural}}[A]=S_{\mathrm{\natural}}[\operatorname{Clos} A]$, there exist $x \in \operatorname{Clos} A$ with $\left|S_{\natural}(x-\chi)\right|=\operatorname{dist}\left(S_{\natural}(\chi), S_{\natural}[A]\right)$ and $b \in \operatorname{Clos} B$ with $|x-b|=\operatorname{dist}(x, B)$, hence, noting

$$
\begin{gathered}
\left|S_{\natural}(\chi-x)\right| \leq \delta(r), \quad\left|S_{\natural}(x-a)\right| \leq\left|S_{\natural}(x-\chi)\right|+\left|S_{\natural}(\chi-a)\right| \leq 2 r, \\
|x-a| \leq(1+\kappa)\left|S_{\natural}(x-a)\right| \leq 2(1+\kappa) r, \quad|x-b| \leq d((3+2 \kappa) r), \\
\left|S_{\natural}(\chi-b)\right| \leq\left|S_{\natural}(\chi-x)\right|+\left|S_{\natural}(x-b)\right| \leq \delta(r)+|x-b|, \\
|\chi-b| \leq(1+\kappa)\left|S_{\natural}(\chi-b)\right|,
\end{gathered}
$$

where $d(s)=\sup \operatorname{dist}(\cdot, B)[A \cap \mathbf{B}(a, s)]$ for $0<s<\infty$, one estimates

$$
|\chi-x| \leq|\chi-b|+|b-x| \leq(2+\kappa)(\delta(r)+d((3+2 \kappa) r)) .
$$

Therefore, in view of $3.2, B$ satisfies (1) and the first implication is proven.

Secondly, if $f: S \rightarrow S^{\perp}$ is of class $\gamma$ and $B=\{\chi+f(\chi): \chi \in S\}$ satisfies (1), then $f$ satisfies (3). For this purpose choose $r>0$ and $0 \leq \lambda<\infty$ such that

$$
\operatorname{Lip}\left(f \mid \mathbf{B}\left(S_{\natural}(a),(2+\kappa) r\right)\right) \leq \lambda .
$$

If $\chi \in B$ and $\left|S_{\natural}(\chi-a)\right| \leq s \leq r$, then there exists $x \in$ Clos $A$ with $|\chi-x|=$ $\operatorname{dist}(\chi, A)$ and, noting $S_{\natural}(x) \in \operatorname{Clos} S_{\natural}[A]$ and $|\chi-a| \leq(1+\lambda) s$, one infers

$\left.\operatorname{dist}\left(S_{\natural}(\chi), S_{\natural}[A]\right) \leq\left|S_{\natural}(\chi-x)\right| \leq|\chi-x| \leq \sup \operatorname{dist}(\cdot, A)[B \cap \mathbf{B}(a,(1+\lambda) s))\right]$, hence, noting $S_{\natural}[B]=S$, the set $S_{\natural}[A]$ is strongly pointwise differentiable of order 1 [order $\gamma]$ at $S_{\natural}(a) \in \operatorname{Clos} S_{\natural}[A]$ and

$$
\operatorname{Tan}\left(S_{\natural}[A], S_{\natural}(a)\right)=S
$$

by 3.2 and 3.4 Moreover, if $\chi \in A$ and $\left|S_{\natural}(\chi-a)\right| \leq s \leq r$, then $|\chi-a| \leq(1+\kappa) s$ and there exists $b \in B$ such that

$$
|\chi-b|=\operatorname{dist}(\chi, B) \leq \sup \operatorname{dist}(\cdot, B)[A \cap \mathbf{B}(a,(1+\kappa) s)] \leq(1+\kappa) r,
$$

hence, defining $x=S_{\natural}(\chi)+f\left(S_{\natural}(\chi)\right)$ and noting $S_{\natural}(x)=S_{\natural}(\chi)$, one obtains

$$
\begin{gathered}
\left|S_{\natural}(b-a)\right| \leq\left|S_{\natural}(\chi-b)\right|+\left|S_{\natural}(\chi-a)\right| \leq(2+\kappa) r, \\
x \in B, \quad|x-b| \leq(1+\lambda)\left|S_{\natural}(x-b)\right| \leq(1+\lambda)|\chi-b|, \\
\left|S_{\natural}^{\perp}(\chi)-f\left(S_{\natural}(\chi)\right)\right|=|\chi-x| \leq|\chi-b|+|x-b| \leq(2+\lambda)|\chi-b| .
\end{gathered}
$$


Therefore $f$ satisfies (3) and the second implication is proven.

In view of the first implication with $A, a$, and $B$ replaced by $S_{\natural}[A], S_{\natural}(a)$, and $S$, the uniqueness of $P$ and $\sqrt{6}$ follow from 2.3 and 2.6 . Therefore the preceding two implications and the initial paragraph yield the equivalence of $(1)-(3)$ and (6) implies (4) and (5).

3.12 Definition. Suppose $n$ is a positive integer and $A \subset \mathbf{R}^{n}$.

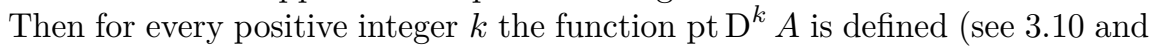
3.11 to be the function whose domain consists of all $(a, S)$ such that $a \in \operatorname{Clos} A$, the set $A$ is pointwise differentiable of order $k$ at $a$, and

$$
S \in \mathbf{G}(n, \operatorname{dim} \operatorname{Tan}(A, a)), \quad S^{\perp} \cap \operatorname{Tan}(A, a)=\{0\}
$$

and whose value at such $(a, S)$ equals the unique $\phi \in \bigodot^{k}\left(\mathbf{R}^{n}, \mathbf{R}^{n}\right)$ such that for some function $f: S \rightarrow S^{\perp}$ of class $k$ there holds $\phi=\mathrm{D}^{k}\left(f \circ S_{\natural}\right)(a)$ and

$$
\begin{gathered}
\lim _{r \rightarrow 0+} r^{-1} \sup |\operatorname{dist}(\cdot, A)-\operatorname{dist}(\cdot, B)|[\mathbf{B}(a, r)]=0, \\
\lim _{r \rightarrow 0+} r^{-k} \sup \operatorname{dist}(\cdot, B)[A \cap \mathbf{B}(a, r)]=0,
\end{gathered}
$$

where $B=\{\chi+f(\chi): \chi \in S\}$. Abbreviate pt $\mathrm{D}^{1} A=\operatorname{ptD} A$. Moreover, the function pt $\mathrm{D}^{0} A$ is defined to be the function whose domain consists of all $(a, S) \in(\operatorname{Clos} A) \times \bigcup_{m=0}^{n} \mathbf{G}(n, m)$ and whose value at such $(a, S)$ equals $S_{\natural}^{\perp}(a)$. The value pt $\mathrm{D}^{i} A(a, S)$ is called the pointwise differential of order $i$ of $A$ at $(a, S)$ whenever $i$ is a nonnegative integer and $(a, S) \in \operatorname{dmn} \operatorname{ptD}^{i} A .19$

3.13 Remark. If $k$ is a positive integer and $(a, S) \in \mathrm{dmn} \mathrm{pt}^{k} A$, then, by 3.11 (4), the values $\mathrm{pt}^{i} A(a, S)$ for $i=0, \ldots, k$ determine $\operatorname{pt~}^{i} A(a, \cdot)$ for $i=0, \ldots, k$.

3.14 Corollary. Suppose $k$ and $n$ are positive integers, $m$ is an integer, $0 \leq$ $m \leq n 0 \leq \alpha \leq 1, \gamma=k$ if $\alpha=0$ and $\gamma=(k, \alpha)$ if $\alpha>0, S \in \mathbf{G}(n, m)$, $x \in X \subset S, f: X \rightarrow S^{\perp}, f$ is continuous at $x, A=\{\chi+f(\chi): \chi \in \operatorname{dmn} f\}$, and $a=x+f(x)$.

Then the following two conditions are equivalent.

(1) The set $A$ is pointwise [strongly pointwise] differentiable of order $\gamma$ at a, $\operatorname{Tan}(A, a) \in \mathbf{G}(n, m)$, and $S^{\perp} \cap \operatorname{Tan}(A, a)=\{0\}$.

(2) The set $X$ is strongly pointwise differentiable of order 1 [order $\gamma$ ] at $x$, $\operatorname{Tan}(X, x)=S$, and there exists a polynomial function $P: S \rightarrow S^{\perp}$ of degree at most $k$ satisfying

$$
\begin{gathered}
\lim _{r \rightarrow 0+} r^{-k} \sup |f-P|[X \cap \mathbf{B}(x, r)]=0 \quad \text { if } \alpha=0, \\
\limsup r^{-k-\alpha} \sup |f-P|[X \cap \mathbf{B}(x, r)]<\infty \quad \text { if } \alpha>0 .
\end{gathered}
$$

\footnotetext{
${ }^{19}$ Anticipating the results of this paper and its logical sequel [San17], we remark that - employing the terminology of approximate differentiation from San17, 3.8, 3.19] - the following proposition may be deduced from [San17, 4.1, 4.3, 4.11] and 3.10 3.11], (4): Whenever $a \in \mathbf{R}^{n}, A \subset \mathbf{R}^{n}, k$ is a positive integer, $0 \leq \alpha \leq 1, \gamma=k$ if $\alpha=0$ and $\gamma=(k, \alpha)$ if $\alpha>0$ $A$ is approximately differentiable of order 1 at $a, T=\operatorname{ap} \operatorname{Tan}(A, a)$, and $m=\operatorname{dim} T \geq 1$, the following two conditions are equivalent:

(1) The set $A$ is approximately differentiable of order $(k, \alpha)$ at a.

(2) There exists a subset $B$ of $\mathbf{R}^{n}$ that is pointwise differentiable of order $\gamma$ at a and satisfies the conditions $\operatorname{Tan}(B, a)=T$ and $\Theta^{m}\left(\mathscr{H}^{m}\llcorner A \sim B, a)=0\right.$.
}

In this case, ap $\mathrm{D}^{i} A(a)=\operatorname{ptD}^{i} B(a, T)$ for $i=2, \ldots, k$. In $(2)$, one may require $B \subset A$. 
In this case $\mathrm{D}^{i}\left(P \circ S_{\sharp}\right)(a)=\mathrm{ptD}^{i} A(a, S)$ for $i=0, \ldots, k$.

Proof. As (1) implies the existence of $r>0,0 \leq \kappa<\infty$, and $s>0$ such that

$A \cap \mathbf{B}(a, r) \subset\left\{\chi:\left|S_{\natural}^{\perp}(\chi-a)\right| \leq \kappa\left|S_{\natural}(\chi-a)\right|\right\}, \quad A \cap S_{\natural}^{-1}\left[\mathbf{B}\left(S_{\natural}(a), s\right)\right] \subset \mathbf{B}(a, r)$

by 3.10 and the continuity of $f$ at $x$ respectively, the equivalence and the postscript follow from 3.4 and 3.11 (4) (6).

3.15 Remark. If $X$ is a neighbourhood of $x$ in $S$, then 2 is equivalent to the requirement that $f$ is pointwise differentiable of order $\gamma$ at $x$.

3.16 Remark. In view of 3.10 and 3.11 (6), it is clear that if $f$ is not continuous at $x$ and $0<m<n$ it may happen that (1) is satisfied but $\lim _{\sup _{\chi \rightarrow x}}|f(\chi)|=\infty$ in which case 22 does not hold.

3.17 Example. If $A$ is an $m+1$ dimensional convex subset of $\mathbf{R}^{n}$, then the relative boundary of $A$ is strongly pointwise differentiable of order 2 at $\mathscr{H}^{m}$ almost all of its points; in fact, one may employ Alexandrov's theorem, see for instance [EG15, Theorem 6.9], to deduce this from 3.14 and 3.15

3.18 Lemma. Suppose $k$ and $n$ are positive integers, $m$ is an integer, $0 \leq m \leq n$, $S \in \mathbf{G}(n, m), A \subset \mathbf{R}^{n}, P: S \rightarrow S^{\perp}$ is a homogeneous polynomial function of degree $k, B=\{\chi+P(\chi): \chi \in S\}$, and $\beta_{s}: \mathbf{R}^{n} \rightarrow \mathbf{R}^{n}$ satisfy

$$
\beta_{s}(x)=s^{-1} S_{\natural}(x)+s^{-k} S_{\natural}^{\perp}(x) \quad \text { for } x \in \mathbf{R}^{n} \text { and } 0<s<\infty .
$$

Then the following two conditions are equivalent.

(1) The set $A$ is pointwise differentiable of order $k$ at 0 and

$$
\operatorname{ptD}^{i} A(0, S)=\mathrm{D}^{i}\left(P \circ S_{\natural}\right)(0) \quad \text { for } i=0, \ldots, k .
$$

(2) There holds

$$
\lim _{s \rightarrow 0+} \operatorname{dist}\left(x, \beta_{s}[A]\right)=\operatorname{dist}(x, B) \quad \text { for } x \in \mathbf{R}^{n} .
$$

Proof. Notice that $\beta_{s}^{-1}=\beta_{1 / s}$ and $\beta_{s}[B]=B$ for $0<s<\infty$ and

$$
\operatorname{Lip} \beta_{s} \leq s^{-k}, \quad \beta_{1 / s}[\mathbf{B}(0, r)] \subset \mathbf{B}(0, s r), \quad \mathbf{B}(0, r / s) \subset \beta_{s}[\mathbf{B}(0, r)],
$$

whenever $0<s \leq 1$ and $0<r<\infty$, in particular

$$
\begin{gathered}
\operatorname{dist}\left(\beta_{s}(a), B\right) \leq s^{-k} \operatorname{dist}(a, B) \quad \text { for } a \in A, \\
\sup \operatorname{dist}(\cdot, B)\left[\beta_{s}[A] \cap \mathbf{B}(0, r)\right] \leq s^{-k} \sup \operatorname{dist}(\cdot, B)[A \cap \mathbf{B}(0, s r)] .
\end{gathered}
$$

Suppose (1) holds. To prove (2), one may assume

$$
A \subset\left\{\chi:\left|S_{\natural}^{\perp}(\chi)\right| \leq \kappa\left|S_{\natural}(\chi)\right|\right\} \quad \text { for some } 0 \leq \kappa<\infty
$$

by 3.4 and 3.10 Whenever $0<r<\infty$ one estimates

$$
\begin{aligned}
\sup \operatorname{dist}\left(\cdot, \beta_{s}[A]\right)[B \cap & \mathbf{B}(0, r)] \leq \lambda s^{-1} \sup \operatorname{dist}\left(\cdot, S_{\natural}[A]\right)[S \cap \mathbf{B}(0, s r)] \\
& +s^{-k} \sup \left\{\left|S_{\natural}^{\perp}(a)-P\left(S_{\natural}(a)\right)\right|: a \in A,\left|S_{\natural}(a)\right| \leq 2 s r\right\}
\end{aligned}
$$


for $0<s \leq 1$, where $\lambda=1+\operatorname{Lip}(P \mid \mathbf{B}(0,2 r))$; in fact, it is sufficient to notice

$$
\begin{gathered}
b-\beta_{s}(a)=S_{\natural}\left(b-s^{-1} a\right)+P\left(S_{\natural}(b)\right)-P\left(S_{\natural}\left(s^{-1} a\right)\right)+s^{-k}\left(P\left(S_{\natural}(a)\right)-S_{\natural}^{\perp}(a)\right), \\
\left|b-\beta_{s}(a)\right| \leq \lambda s^{-1}\left|S_{\natural}(s b)-S_{\natural}(a)\right|+s^{-k}\left|S_{\natural}^{\perp}(a)-P\left(S_{\natural}(a)\right)\right|
\end{gathered}
$$

whenever $b \in B \cap \mathbf{B}(0, r), a \in A$, and $\left|S_{\natural}(a)\right| \leq 2 s r$, as $0 \in \operatorname{Clos} A$ by 3.4 implies

$$
0 \in \operatorname{Clos} S_{\natural}[A], \quad \operatorname{dist}\left(S_{\natural}(s b), S_{\natural}[A] \cap \mathbf{B}(0,2 s r)\right)=\operatorname{dist}\left(S_{\natural}(s b), S_{\natural}[A]\right) .
$$

In conjunction with the first paragraph, (2) now follows using 3.2 and 3.116 .

Conversely, suppose (2) holds. In order to prove (1), firstly notice that $0 \in \operatorname{Clos} A$ as $0 \in B$. Next, the following assertion will be shown:

$$
\begin{gathered}
A \cap \mathbf{B}(0, r) \subset\left\{\chi:\left|S_{\natural}^{\perp}(\chi)\right| \leq \kappa\left|S_{\natural}(\chi)\right|\right\} \quad \text { for some } r>0 \text { and } 0 \leq \kappa<\infty, \\
\lim _{A \ni x \rightarrow 0}\left|S_{\natural}^{\perp}(x)-P\left(S_{\natural}(x)\right)\right| /\left|S_{\natural}(x)\right|^{k}=0 .
\end{gathered}
$$

For this purpose let $\varepsilon>0$ and $C=\mathbf{R}^{n} \cap\left\{x:\left|S_{\natural}^{\perp}(x)-P\left(S_{\natural}(x)\right)\right| \geq \varepsilon\left|S_{\natural}(x)\right|^{k}\right\}$. Clearly, $B \cap C=\{0\}$ and $\beta_{s}[C]=C$ for $0<s<\infty$. Since, by [Fed69, 2.10.21],

$$
\operatorname{dist}\left(\cdot, \beta_{s}[A]\right) \rightarrow \operatorname{dist}(\cdot, B) \quad \text { uniformly on compact subsets of } \mathbf{R}^{n} \text { as } s \rightarrow 0+
$$

there exists $0<s<\infty$ with $\beta_{t}[A] \cap C \cap\{x:|x|=1\}=\varnothing$ for $0<t \leq s$, hence

$$
A \cap \beta_{1 / s}[\mathbf{B}(0,1)] \cap C \subset\{0\}
$$

as $\beta_{1 / s}[\mathbf{B}(0,1)] \sim\{0\}=\bigcup\left\{\beta_{1 / t}\left[\mathbf{R}^{n} \cap\{x:|x|=1\}\right]: 0<t \leq s\right\}$, and the assertion follows. In particular, one may assume $A \subset\left\{\chi:\left|S_{\natural}^{\perp}(\chi)\right| \leq \kappa\left|S_{\natural}(\chi)\right|\right\}$. Noting

$$
\begin{gathered}
s^{-1} \operatorname{dist}\left(S_{\natural}(s x), S_{\natural}[A]\right) \leq\left|S_{\natural}\left(x-\beta_{s}(a)\right)\right| \leq\left|x-\beta_{s}(a)\right| \quad \text { for } a \in A, x \in \mathbf{R}^{n}, \\
s^{-1} \sup \operatorname{dist}\left(\cdot, S_{\natural}[A]\right)[S \cap \mathbf{B}(0, s)] \leq \sup \operatorname{dist}\left(\cdot, \beta_{s}[A]\right)\left[B \cap S_{\natural}^{-1}[\mathbf{B}(0,1)]\right]
\end{gathered}
$$

for $0<s<\infty$, the set $S_{\natural}[A]$ is strongly pointwise differentiable of order 1 at 0 with $\operatorname{Tan}\left(S_{\natural}[A], 0\right)=S$ by 3.2 and 3.4 . Consequently, 3.11 (6) yields (1).

3.19 Remark. It follows by 3.4 3.10, and 3.11, 4 that a subset $A$ of $\mathbf{R}^{n}$ is pointwise differentiable of order 1 at $a$ if and only if $A_{s}=\mathbf{R}^{n} \cap\{x: a+s x \in A\}$ satisfy

$$
\lim _{s \rightarrow 0+} \operatorname{dist}\left(x, A_{s}\right)=\operatorname{dist}(x, T) \quad \text { whenever } x \in \mathbf{R}^{n}
$$

for some subspace $T$ of $\mathbf{R}^{n}$. In this case $T=\operatorname{Tan}(A, a)$. (But this condition on $\operatorname{Tan}(A, a)$ does not imply pointwise differentiability of order 1 of $A$ at $a$.)

3.20. Suppose $n$ is a positive integer, $m$ is an integer, $0 \leq m \leq n, S \in \mathbf{G}(n, m)$, $T \subset \mathbf{R}^{n}, h \in \operatorname{Hom}\left(S, S^{\perp}\right)$, and $L=\mathbf{1}_{\mathbf{R}^{n}}-h \circ S_{\mathfrak{t}}$. Then $S^{\perp} \cap T=\{0\}$ if and only if $S^{\perp} \cap L[T]=\{0\}$.

3.21 Lemma. Suppose $k$ and $n$ are positive integers, $m$ is an integer, $0 \leq m \leq n$, $A \subset \mathbf{R}^{n}, S \in \mathbf{G}(n, m), f: S \rightarrow S^{\perp}$ of class $k, B=\left\{x-f\left(S_{\natural}(x)\right): x \in A\right\}$, $a \in \mathbf{R}^{n}$, and $b=a-f\left(S_{\sharp}(a)\right)$.

Then the following two statements are equivalent.

(1) The set $A$ is pointwise differentiable of order $k$ at $a, \operatorname{Tan}(A, a) \in \mathbf{G}(n, m)$, and $S^{\perp} \cap \operatorname{Tan}(A, a)=\{0\}$. 
(2) The set $B$ is pointwise differentiable of order $k$ at $b, \operatorname{Tan}(B, b) \in \mathbf{G}(n, m)$, and $S^{\perp} \cap \operatorname{Tan}(B, a)=\{0\}$.

In this case $\mathrm{pt} \mathrm{D}^{i} A(a, S)=\mathrm{pt}^{i} B(b, S)+\mathrm{D}^{i}\left(f \circ S_{\natural}\right)(a)$ for $i=0, \ldots, k$.

Proof. Define $g: \mathbf{R}^{n} \rightarrow \mathbf{R}^{n}$ by $g(x)=x-f\left(S_{\natural}(x)\right)$ for $x \in \mathbf{R}^{n}$. Noting $\operatorname{Tan}(B, b)=\mathrm{D} g(a)[\operatorname{Tan}(A, a)]$ by Fed69, 3.1.21, the principal conclusion follows from 3.7 and 3.20 The postscript then is a consequence of 3.10 and 3.11 (6).

3.22 Theorem. Suppose $k$ and $n$ are positive integers, $m$ is an integer with $0 \leq m \leq n, a \in \mathbf{R}^{n}, A \subset \mathbf{R}^{n}$, the set $A$ is pointwise differentiable of order 1 at $a$, $S \in \mathbf{G}(n, m)$, and $P_{1} \in \operatorname{Hom}\left(S, S^{\perp}\right)$ satisfies $\operatorname{Tan}(A, a)=\left\{\chi+P_{1}(\chi): \chi \in S\right\}$.

Then the following two conditions are equivalent.

(1) The set $A$ is pointwise differentiable of order $k$ at a.

(2) There exist homogeneous polynomial functions $P_{i}: S \rightarrow S^{\perp}$ of degree $i$ for $i=2, \ldots, k$ such that the following condition holds: If

$$
A_{1}=\{x-a: x \in A\}, \quad A_{i}=\left\{x-P_{i-1}\left(S_{\natural}(x)\right): x \in A_{i-1}\right\}
$$

for $i=2, \ldots, k$, then $B_{i}=\left\{\chi+P_{i}(\chi): \chi \in S\right\}$ satisfy

$$
\lim _{s \rightarrow 0+} \operatorname{dist}\left(x, \beta_{i, s}\left[A_{i}\right]\right)=\operatorname{dist}\left(x, B_{i}\right) \quad \text { for } x \in \mathbf{R}^{n} \text { and } i=2, \ldots, k,
$$

where $\beta_{i, s}: \mathbf{R}^{n} \rightarrow \mathbf{R}^{n}$ and $\beta_{i, s}(x)=s^{-1} S_{\natural}(x)+s^{-i} S_{\natural}^{\perp}(x)$ for $x \in \mathbf{R}^{n}$.

In this case $\mathrm{pt}^{i} A(a, S)=\mathrm{D}^{i}\left(P_{i} \circ S_{\natural}\right)(0)$ for $i=1, \ldots, k$.

Proof. Assume $a=0$. Notice that pt D $A(0, S)=\mathrm{D}\left(P_{1} \circ S_{\natural}\right)(0)$ by 3.18 and 3.19

Suppose (1) holds and define $P_{i}: S \rightarrow S^{\perp}$ by

$$
P_{i}(\chi)=\left\langle\chi^{i} / i !, \operatorname{ptD}^{i} A(0, S)\right\rangle \quad \text { for } \chi \in S \text { and } i=2, \ldots, k .
$$

Then 3.21 inductively implies that the sets $A_{i}$ are pointwise differentiable of order $k$ at 0 and

$$
\operatorname{ptD}^{j} A_{i}(0, S)=\sum_{l=i}^{k} \mathrm{D}^{j}\left(P_{l} \circ S_{\natural}\right)(0) \quad \text { for } i=1, \ldots, k \text { and } j=0, \ldots, k \text {, }
$$

hence applying 3.18 with $k, A$, and $P$ replaced by $i, A_{i}$, and $P_{i}$ yields $(2)$.

Now, suppose $(2)$ holds. Then $A_{i}$ is pointwise differentiable of order $i$ and

$$
\operatorname{ptD}^{j} A_{i}(0, S)=\mathrm{D}^{j}\left(P_{i} \circ S_{\natural}\right)(0) \text { for } j=0, \ldots, i \text { and } i=1, \ldots, k
$$

by 3.18 hence 3.21 yields (1) and the postscript.

3.23 Remark. The convergence in (2) is equivalent (by 3.2 and [Fed69, 2.10.21]) to "convergence locally in Hausdorff distance" of Clos $\beta_{i, s}\left[A_{i}\right]$ to $B_{i}$ as $s \rightarrow 0+$, see David [Dav03, 3.1] for a definition in the case of sequences of closed sets, and to "Kuratowski convergence" of the same sets, see Beer [Bee85. Theorem 1]. 


\section{Higher order differentiability theory for func- tions}

This section provides the main differentiability theorem for functions in 4.6 which serves as a model for the case of sets treated in Section 5 In fact, the theorems employed in its proof, 4.1, 4.2 and 4.4 will also be used to treat the case of sets.

4.1 Theorem (classical). Suppose $X$ and $Y$ are complete, separable metric spaces, and $f$ is a function mapping a subset of $X$ into $Y$.

Then the following two statements are equivalent.

(1) The domain of $f$ is a Borel subset of $X$ and $f$ is a Borel function.

(2) The set $f$ is a Borel subset of $X \times Y$.

Proof. Defining $p: X \times Y \rightarrow X$ and $q: X \times Y$ by $p(x, y)=x$ and $q(x, y)=y$ for $(x, y) \in X \times Y$, one notices that $f^{-1}[B]=p\left[f \cap q^{-1}[B]\right]$ for $B \subset Y$, hence (2) implies (1) by Fed69, 2.2.10] as $p \mid f$ is univalent. The converse is elementary.

4.2 Theorem. Suppose $k$ is a nonnegative integer, $m$ and $n$ are positive integers, $m<n, 0 \leq \alpha \leq 1, \gamma=k$ if $\alpha=0$ and $\gamma=(k, \alpha)$ if $\alpha>0, D$ is $\mathscr{L}^{m}$ measurable, $f_{i}: D \rightarrow \bigodot^{i}\left(\mathbf{R}^{m}, \mathbf{R}^{n-m}\right)$ are $\mathscr{L}^{m}\llcorner D$ measurable for $i=0, \ldots, k$, and $\mathscr{L}^{m}$ almost all $a \in D$ satisfy

$$
\begin{gathered}
\lim _{r \rightarrow 0+} r^{-k} \sup \left|f-P_{a}\right|[D \cap \mathbf{B}(a, r)]=0 \quad \text { if } \alpha=0, \\
\limsup _{r \rightarrow 0+} r^{-k-\alpha} \sup \left|f-P_{a}\right|[D \cap \mathbf{B}(a, r)]<\infty \quad \text { if } \alpha>0,
\end{gathered}
$$

where $P_{a}: \mathbf{R}^{m} \rightarrow \mathbf{R}^{n-m}$ and $P_{a}(x)=\sum_{i=0}^{k}\left\langle(x-a)^{i} / i !, f_{i}(a)\right\rangle$ for $x \in \mathbf{R}^{m}$.

Then there exists a sequence of functions $g_{j}: \mathbf{R}^{m} \rightarrow \mathbf{R}^{n-m}$ of class $\gamma$ such that $\mathscr{L}^{m}\left(D \sim \bigcup_{j=1}^{\infty}\left\{x: f_{0}(x)=g_{j}(x)\right\}\right)=0$.

Proof. Assuming $D$ to be a Borel subset of $\mathbf{R}^{m}$ with $\mathscr{L}^{m}(D)<\infty$ and noting [Fed69, 2.10.19(4)], this follows from Isakov [Isa87a, Theorem 1] if $\alpha=0$ and Isakov Isa87a, Theorem 2] and 2.5 if $\alpha>0$.

4.3 Remark. Isakov in fact provides a characterisation of the property in the conclusion (called " $\mathscr{C}^{k+\alpha}$-rectifiability" by Anzellotti and Serapioni in AS94, $2.4]$ ) in terms of "approximate differentiability of the jet $f_{i}, i=0, \ldots, k$ ", see Isa87a. A further characterisation using approximate partial derivatives of higher order is given by Lin and Liu [LL13, 1.5].

4.4 Theorem. Suppose $g: \mathbf{R}^{m} \rightarrow\{t: 0 \leq t \leq \infty\}$ and $0<l<\infty$.

Then $\mathscr{L}^{m}$ almost all a satisfy that

$$
\limsup _{r \rightarrow 0+} r^{-l} \sup g[\mathbf{U}(a, r)] \quad \text { equals either } 0 \text { or } \infty \text {. }
$$

${ }^{20}$ As the proof of [Isa87a Theorem 2] is omitted in that reference as "completely analogous" to Isa87a Theorem 1], the reader may find it helpful to notice that the presently needed case of [Isa87a, Theorem 2] is in fact simpler than Isa87a, Theorem 1] provided one refers to [Ste70 VI.2.2.2, VI.2.3.1-3] instead of Fed69 3.1.14 for the Whitney type extension theorem. 
Proof. Define closed sets $E_{i}=\mathbf{R}^{m} \cap\left\{y: \sup g[\mathbf{U}(y, r)] \leq i r^{l}\right.$ for $\left.0<r<1 / i\right\}$ for every positive integer $i$. It will be sufficient to show

$$
\lim _{r \rightarrow 0+} r^{-l} \sup g[\mathbf{U}(a, r)]=0 \quad \text { for } \mathscr{L}^{m} \text { almost all } a \in E_{i} \text {. }
$$

Suppose $a \in E_{i}$ and $0<r<1 / i$.

Whenever $x \in \mathbf{U}(a, r)$ one chooses $y \in E_{i}$ with $|x-y|=\operatorname{dist}\left(x, E_{i}\right)$ and infers that $|x-y| \leq|x-a|<1 / i$ and $g(x) \leq i|y-x|^{l}$. This implies

$$
r^{-l} \sup g[\mathbf{U}(a, r)] \leq i\left(r^{-1} \sup \operatorname{dist}\left(\cdot, E_{i}\right)[\mathbf{U}(a, r)]\right)^{l} .
$$

As $r$ approaches 0 the right hand side of the preceding inequality approaches 0 provided $E_{i}$ is pointwise differentiable of order 1 at $a$ and $\operatorname{Tan}\left(E_{i}, a\right)=\mathbf{R}^{m}$ which is true at $\mathscr{L}^{m}$ almost all $a \in E_{i}$ by 3.9

4.5 Remark. Taking $n=m, U=\mathbf{R}^{m}, V$ the varifold associated to $\mathscr{L}^{m}$, and $q=l$, this is a special case of Kolasiński and the author [KM17, 4.4]. The proof is included for the convenience of the reader and is modelled on [Fed69, 2.9.17]. A similar result with certain Lebesgue seminorms of $g$ replacing "sup $g[\mathbf{U}(a, r)]$ " was obtained by Calderón and Zygmund in [CZ61, Theorem 10 (ii)]. Further variants and comments on the history and the sharpness of general results of this type are contained in [Men09, 3.1-3.4] and [KM17, 4.1-4.5].

4.6 Theorem. Suppose $k$ is a nonnegative integer, $m$ and $n$ are positive integers, $m<n, 0 \leq \alpha \leq 1, \gamma=k$ if $\alpha=0$ and $\gamma=(k, \alpha)$ if $\alpha>0, U$ is an open subset of $\mathbf{R}^{m}, f: U \rightarrow \mathbf{R}^{n-m}$, and $X$ is the set of $x$ at which $f$ is pointwise differentiable of order $\gamma$.

Then the following four statements hold.

(1) The functions $\mathrm{pt}^{i} f$ are Borel functions whose domains are Borel subsets of $\mathbf{R}^{m}$ for $i=0, \ldots, k$ and $X$ is a Borel subset of $\mathbf{R}^{m}$.

(2) There exists a sequence of functions $g_{j}: \mathbf{R}^{m} \rightarrow \mathbf{R}^{n-m}$ of class $\gamma$ such that $\mathscr{L}^{m}\left(X \sim \bigcup_{j=1}^{\infty}\left\{x: f(x)=g_{j}(x)\right\}\right)=0$.

(3) If $g: \mathbf{R}^{m} \rightarrow \mathbf{R}^{n-m}$ is of class $\gamma$ and $Y=\{y: f(y)=g(y)\}$, then

$$
\begin{gathered}
\operatorname{pt}^{i} f(a)=\mathrm{D}^{i} g(a) \quad \text { for } i=0, \ldots, k, \\
\lim _{x \rightarrow a}|f(x)-g(x)| /|x-a|^{k+\alpha}=0
\end{gathered}
$$

at $\mathscr{L}^{m}$ almost all $a \in X \cap Y$.

(4) If $\alpha=1$, then $f$ is pointwise differentiable of order $k+1$ at $\mathscr{L}^{m}$ almost all $a \in X$.

Proof. Assume $U=\mathbf{R}^{m}$.

Abbreviate $C=\mathbf{R}^{m} \times \prod_{l=0}^{k} \bigodot^{l}\left(\mathbf{R}^{m}, \mathbf{R}^{n-m}\right)$. Whenever $i$ and $j$ are positive integers define $D_{i, j}$ to consist of those $\left(a, \phi_{0}, \ldots, \phi_{k}\right) \in C$ satisfying

$$
\left|f(x)-\sum_{l=0}^{k}\left\langle(x-a)^{l} / l !, \phi_{l}\right\rangle\right| \leq|x-a|^{k} / i \quad \text { for } x \in \mathbf{U}(a, 1 / j)
$$

and $E_{i}$ to consist of those $\left(a, \phi_{0}, \ldots, \phi_{k}\right) \in C$ satisfying

$$
\left|f(x)-\sum_{l=0}^{k}\left\langle(x-a)^{l} / l !, \phi_{l}\right\rangle\right| \leq i|x-a|^{k+\alpha} \quad \text { for } x \in \mathbf{U}(a, 1 / i) .
$$


Furthermore, define

$$
F=\left\{\left(a, \operatorname{ptD}^{0} f(a), \ldots, \operatorname{pt} \mathrm{D}^{k} f(a)\right): a \in \operatorname{dmn} \operatorname{ptD}^{k} f \text { and } a \in X\right\} .
$$

Since the sets $D_{i, j}$ and $E_{i}$ are closed and

$$
F=\bigcap_{i=1}^{\infty} \bigcup_{j=1}^{\infty} C_{i, j} \quad \text { if } \alpha=0, \quad F=\bigcup_{i=1}^{\infty} E_{i} \quad \text { if } \alpha>0
$$

$F$ is a Borel set. Noting that the condition $a \in X$ in the definition of $F$ is redundant if $\alpha=0$, one infers that $\operatorname{pt~}^{k} f$ is a Borel function whose domain is a Borel set from 4.1. hence the same holds for $\mathrm{pt}^{i} f$ for $i=0, \ldots, k-1$. As

$$
X=\left\{a:\left(a, \operatorname{pt}^{0} f(a), \ldots, \operatorname{pt} \mathrm{D}^{k} f(a)\right) \in F\right\},
$$

$X$ is a Borel set and (1) follows.

(1) and 4.2 yield (2). To prove the first half of (3), it is sufficient to apply 2.3 with $S, Y, \mathrm{D}^{i} P(a)$, and $X$ replaced by $\mathbf{R}^{m}, \mathbf{R}^{n-m}$, pt $\mathrm{D}(f-g)(a)$, and $Y$ for $i=0, \ldots, k$ whenever $a \in X$, the set $Y$ is pointwise differentiable of order 1 at $a$, and $\operatorname{Tan}(Y, a)=\mathbf{R}^{m}$ by 3.9 To deduce the second half of $(3)$ from its first half, one may assume $\alpha>0$ and hence apply 4.4 with $g$ and $l$ replaced by $|f-g|$ and $k+\alpha$. To prove (4), notice that in this case the functions $g_{j}$ in (3) may be required to be of class $k+1$ by [Fed69, 3.1.15, hence (3) implies (4).

4.7 Remark. In view of 2.8 , the case $k=0$ of (1) merely restates that the set of continuity points of $f$ is a Borel set, see for instance [HS75, (6.90) (a)-(c)].

4.8 Remark. The proof of (1) is a variation of the argument of [Fed69, 3.1.1 where the case $k=1$ is treated for continuous $f$. The case $k=1$ for arbitrary $f$ occurs in Járai Jár85. In fact, it is shown there that $(\mathrm{D} f)^{-1}[C]$ belongs to the class " $\mathscr{F}_{\sigma \delta}$ " whenever $C$ is a closed subset of $\operatorname{Hom}\left(\mathbf{R}^{m}, \mathbf{R}^{n-m}\right)$. That class is also denoted " $\boldsymbol{\Pi}_{3}^{0}\left(\mathbf{R}^{m}\right)$ " within the Borel hierarchy, see [Kec95].

4.9 Remark. The pattern proof of (2)-4 is that of Liu [Liu08, 1.6].

4.10 Remark. A related study of pointwise differentials of order $k$ in terms of general difference quotients is carried out by Zibman, see [Zib78].

\section{$5 \quad$ Higher order differentiability theory for sets}

In this section Theorem $\mathrm{B}$ is proven, see 5.2 and 5.5 for the Borel properties asserted in $B$ (1) and 5.7 for the differentiability results asserted in $B(2)-(4)$.

5.1. Suppose $n$ is a positive integer, $m$ is an integer, and $0 \leq m \leq n$. Then the set

$$
(\mathbf{G}(n, m) \times \mathbf{G}(n, m)) \cap\left\{(S, T): S^{\perp} \cap T=\{0\}\right\}
$$

is an open subset of $\mathbf{G}(n, m) \times \mathbf{G}(n, m)$; in fact, $S^{\perp} \cap T=\{0\}$ if and only if $\bigwedge_{m}\left(T_{\natural} \circ S_{\natural}\right) \neq 0$ for $S, T \in \mathbf{G}(n, m)$.

5.2 Theorem. Suppose $A \subset \mathbf{R}^{n}$ and

$$
\tau=\{(a, S): S=\operatorname{Tan}(A, a)\} \cap \operatorname{dmn} \operatorname{pt} \mathrm{D} A \subset \mathbf{R}^{n} \times \bigcup_{m=0}^{n} \mathbf{G}(n, m) .
$$

Then $\tau$ is a Borel function whose domain is a Borel set and $\tau^{-1}[\mathbf{G}(n, m)]$ is countably $m$ rectifiable whenever $m=0, \ldots, n$. 
Proof. Let $V=\mathbf{R}^{n}$ and $G=\bigcup_{m=0}^{n} \mathbf{G}(n, m)$. Endowing $\mathbf{R}^{V}$ with the Cartesian product topology ${ }^{21}$ one notices that $\delta: V \times G \rightarrow \mathbf{R}^{V}$ defined by

$$
\delta(a, S)(x)=\operatorname{dist}(x,\{a+\chi: \chi \in S\}) \quad \text { for }(a, S) \in V \times G \text { and } x \in V
$$

is continuous and defines closed sets $C_{i, j}$ consisting of those $(a, S) \in V \times G$ with

$$
a \in \operatorname{Clos} A, \quad \sup |\operatorname{dist}(\cdot, A)-\delta(a, S)|[\mathbf{U}(a, r)] \leq r / i \quad \text { for } 0<r<1 / j
$$

whenever $i$ and $j$ are positive integers. Observe that

$$
\tau=\bigcap_{i=1}^{\infty} \bigcup_{j=1}^{\infty} C_{i, j},
$$

by 3.43 .10 and 3.11 , hence $\tau$ is a Borel set. Since $\tau$ is a function, it follows that $\tau$ is a Borel function whose domain is a Borel set by 4.1 .

Suppose $m$ is an integer and $0 \leq m \leq n$. Choose a countable dense subset $D$ of $\mathbf{G}(n, m)$, define $E(i, S)$ to consist of $a \in \operatorname{Clos} A$ such that

$$
A \cap \mathbf{U}(a, 1 / i) \subset\left\{\chi:\left|S_{\natural}^{\perp}(\chi-a) \leq i\right| S_{\natural}(\chi-a) \mid\right\}
$$

for $i=1,2,3, \ldots$ and $S \in D$. Noting 3.4 and 3.10 one infers from 5.1 that

$$
\tau^{-1}[\mathbf{G}(n, m)] \subset \bigcup\{E(i, S): i=1,2,3, \ldots \text { and } S \in D\} .
$$

Therefore the set $\tau^{-1}[\mathbf{G}(n, m)]$ is countably $m$ rectifiable by [Fed69, 3.3.5].

5.3 Lemma. Suppose $B \subset \mathbf{R}^{n}$ and $h_{j}$ is a sequence of univalent maps of $\mathbf{R}^{n}$ onto $\mathbf{R}^{n}$ satisfying $\lim _{j \rightarrow \infty} h_{j}(x)=x$ for $x \in \mathbf{R}^{n}$, and

$\lim _{j \rightarrow \infty} \sup \left\{\left|h_{j}^{-1}(\chi)-\chi\right|: \chi \in K\right\}=0 \quad$ whenever $K$ is a compact subset of $\mathbf{R}^{n}$.

Then there holds

$$
\lim _{j \rightarrow \infty} \operatorname{dist}\left(x, h_{j}[B]\right)=\operatorname{dist}(x, B) \quad \text { for } x \in \mathbf{R}^{n} .
$$

Proof. Suppose $x \in \mathbf{R}^{n}$. Clearly, $\lim \sup _{j \rightarrow \infty} \operatorname{dist}\left(x, h_{j}[B]\right) \leq \operatorname{dist}(x, B)$. Moreover, if $\varepsilon>0$ and $r=\operatorname{dist}(x, B) \geq 0, j$ is a positive integer, and

$$
\left|h_{j}^{-1}(\chi)-\chi\right| \leq \varepsilon \quad \text { for } \chi \in \mathbf{B}(x, r) \text {, }
$$

then $\left|h_{j}(b)-x\right| \geq r-\varepsilon$ for $b \in B$, as $\left|h_{j}(b)-x\right| \leq r$ implies $\left|b-h_{j}(b)\right| \leq \varepsilon$. It follows that $\operatorname{dist}(x, B) \leq \liminf _{j \rightarrow \infty} \operatorname{dist}\left(x, h_{j}[B]\right)$.

$\mathbf{5 . 4}$ Lemma. Suppose $k$ and $n$ are positive integers, $G=\bigcup_{m=0}^{n} \mathbf{G}(n, m), V=$ $\mathbf{R}^{n}, C$ is the set of

$$
\left(a, S, \phi_{0}, \ldots, \phi_{k}\right) \in V \times G \times \prod_{i=0}^{k} \bigodot^{i}(V, V)
$$

such that $\phi_{0}=S_{\natural}^{\perp}(a)$ and

$$
\phi_{i}\left(v_{1}, \ldots, v_{i}\right)=\phi_{i}\left(S_{\natural}\left(v_{1}\right), \ldots, S_{\natural}\left(v_{i}\right)\right) \in S^{\perp} \quad \text { for } v_{1}, \ldots, v_{i} \in V, i=1, \ldots, k,
$$

${ }^{21}$ Whenever $X$ and $Y$ are sets $Y^{X}$ denotes set of maps from $X$ into $Y$, see Fed69. p. 669]. 
$\mathbf{R}^{V}$ is endowed with the Cartesian product topology, and $\delta: C \rightarrow \mathbf{R}^{V}$ satisfies

$$
\begin{gathered}
\delta\left(a, S, \phi_{0}, \ldots, \phi_{k}\right)(x)=\operatorname{dist}(x,\{\chi+P(\chi): \chi \in S\}) \\
\text { where } P(\chi)=\sum_{i=0}^{k}\left\langle\left(\chi-S_{\natural}(a)\right)^{i} / i !, \phi_{i}\right\rangle,
\end{gathered}
$$

whenever $\left(a, S, \phi_{0}, \ldots, \phi_{k}\right) \in C$ and $x \in V$.

Then $C$ is a closed subset of $V \times G \times \prod_{i=0}^{k} \bigodot^{i}(V, V)$ and $\delta$ is continuous.

Proof. The first statement is trivial.

To prove the second statement, suppose $\left(a_{j}, S_{j}, \phi_{0, j}, \ldots, \phi_{k, j}\right)$ is a sequence in $C$ converging to $\left(a, S, \phi_{0}, \ldots, \phi_{k}\right) \in C$ as $j \rightarrow \infty, P_{j}$ and $P$ are the associated polynomial functions, and

$$
B_{j}=\left\{\chi+P_{j}(\chi): \chi \in S_{j}\right\}, \quad B=\{\chi+P(\chi): \chi \in S\} .
$$

One may assume that $S_{j} \in \mathbf{G}(n, \operatorname{dim} S)$ for every positive integer $j$. Hence, as $\mathbf{O}(n)$ operates on the homogeneous space $\mathbf{G}(n, \operatorname{dim} S)$ by a transitive left action, see [Fed69, 2.7.1, 3.2.28(2) (4)], there exists a sequence $f_{j} \in \mathbf{O}(n)$ with $f_{j}[S]=S_{j}$ and $f_{j} \rightarrow \mathbf{1}_{V}$ as $j \rightarrow \infty$. Define $g_{j}: V \rightarrow V$ and $g: V \rightarrow V$ by

$$
g_{j}(x)=x-P_{j}\left(\left(S_{j}\right)_{\natural}(x)\right), \quad g(x)=x-P\left(S_{\natural}(x)\right)
$$

whenever $x \in V$ and $j$ is a positive integer and notice that $g_{j}$ map $V$ univalently onto $V$ and

$$
g_{j}^{-1}(x)=x+P_{j}\left(\left(S_{j}\right)_{\natural}(x)\right) \quad \text { for } x \in V .
$$

Since $B_{j}=h_{j}[B]$ for $h_{j}=g_{j}^{-1} \circ f_{j} \circ g$, applying 5.3 yields the conclusion.

5.5 Theorem. Suppose $k$ and $n$ are positive integers, $0 \leq \alpha \leq 1, \gamma=k$ if $\alpha=0$ and $\gamma=(k, \alpha)$ if $\alpha>0, A \subset \mathbf{R}^{n}$, and $X$ is the set of $a \in \mathbf{R}^{n}$ such that $A$ is pointwise [strongly pointwise] differentiable of order $\gamma$ at a.

Then $\mathrm{pt}^{k} A$ is a Borel function whose domain is a Borel set and $X$ is a Borel set.

Proof. Let $C$ and $\delta$ as in 5.4 Whenever $i$ and $j$ are positive integers define $C_{i, j}$ to consist of those $\left(a, S, \phi_{0}, \ldots, \phi_{k}\right) \in C$ with $a \in$ Clos $A$ satisfying

$$
\sup \left|\operatorname{dist}(\cdot, A)-\delta\left(a, S, \phi_{0}, \ldots, \phi_{k}\right)\right|[\mathbf{U}(a, r)] \leq r / i \quad \text { for } 0<r<1 / j,
$$

$D_{i, j}$ to consist of those $\left(a, S, \phi_{0}, \ldots, \phi_{k}\right) \in C$ with $a \in$ Clos $A$ satisfying

$$
\begin{gathered}
\sup \delta\left(a, S, \phi_{0}, \ldots, \phi_{k}\right)[A \cap \mathbf{U}(a, r)] \leq r^{k} / i \quad \text { for } 0<r<1 / j, \\
{\left[\sup \left|\operatorname{dist}(\cdot, A)-\delta\left(a, S, \phi_{0}, \ldots, \phi_{k}\right)\right|[\mathbf{U}(a, r)] \leq r^{k} / i \quad \text { for } 0<r<1 / j,\right]}
\end{gathered}
$$

and $E_{i}$ to consist of those $\left(a, S, \phi_{0}, \ldots, \phi_{k}\right) \in C$ with $a \in$ Clos $A$ satisfying

$$
\begin{gathered}
\sup \delta\left(a, S, \phi_{0}, \ldots, \phi_{k}\right)[A \cap \mathbf{U}(a, r)] \leq i r^{k} \quad \text { for } 0<r<1 / i . \\
{\left[\sup \left|\operatorname{dist}(\cdot, A)-\delta\left(a, S, \phi_{0}, \ldots, \phi_{k}\right)\right|[\mathbf{U}(a, r)] \leq i r^{k} \quad \text { for } 0<r<1 / i .\right]}
\end{gathered}
$$

Furthermore, define

$$
F=\left\{\left(a, S, \operatorname{ptD}^{0} A(a, S), \ldots, \operatorname{ptD}^{k} A(a, S)\right):(a, S) \in \operatorname{dmn} \operatorname{ptD}^{k} A, a \in X\right\}
$$


and notice that the condition $a \in X$ is redundant in the unbracketed case if $\alpha=0$. Since the sets $C_{i, j}, D_{i, j}$, and $E_{i}$ are closed by 5.4 and

$$
F=\bigcap_{i=1}^{\infty} \bigcup_{j=1}^{\infty}\left(C_{i, j} \cap D_{i, j}\right) \quad \text { if } \alpha=0, \quad F=\left(\bigcap_{i=1}^{\infty} \bigcup_{j=1}^{\infty} C_{i, j}\right) \cap \bigcup_{i=1}^{\infty} E_{i} \quad \text { if } \alpha>0
$$

by 3.43 .10 , and 3.11 (4), $F$ is a Borel set. Consequently, 4.1 implies that pt $\mathrm{D}^{i} A$ are Borel functions whose domains are Borel sets for $i=0, \ldots, k$. As

$$
X=\left\{a:\left(a, \tau(a), \operatorname{pt~}^{0} A(a, \tau(a)), \ldots, \operatorname{ptD}^{k} A(a, \tau(a))\right) \in F\right\},
$$

one may use 5.2 to deduce that $X$ is a Borel set.

5.6 Lemma. Suppose $k$ and $n$ are positive integers, $m$ is an integer, $0 \leq m \leq n$, $0 \leq \alpha \leq 1, \gamma=k$ if $\alpha=0$, and $\gamma=(k, \alpha)$ if $\alpha>0, A \subset \mathbf{R}^{n}, a \in \operatorname{Clos} A$, the set $\bar{A}$ is pointwise differentiable of order $\gamma$ at a with $\operatorname{Tan}(A, a) \in \mathbf{G}(n, m)$, $D \subset S \in \mathbf{G}(n, m), f: S \rightarrow S^{\perp}$ is of class $1, x=S_{\natural}(a)$, the set $D$ is pointwise differentiable of order 1 at $x$ with $\operatorname{Tan}(D, x)=S$, and

$$
B=\{\chi+f(\chi): \chi \in D\} \subset \operatorname{Clos} A .
$$

Then $B$ is pointwise differentiable of order $\gamma$ at $a$ and

$$
\operatorname{ptD}^{i} A(a, \cdot)=\operatorname{ptD} D^{i} B(a, \cdot) \quad \text { for } i=0, \ldots, k .
$$

Proof. Possibly replacing $A$ by Clos $A$, one may assume $A$ to be closed, hence $a \in A$ and $B \subset A$. Noting $x \in \operatorname{Clos} D$ by 3.4. one may also assume $x \in D$ possibly replacing $D$ by $D \cup\{x\}$. Define

$$
C=\left\{\chi+\sum_{i=0}^{k}\left\langle(\chi-x)^{i} / i !, \operatorname{ptD}^{i} A(a, S)\right\rangle: \chi \in S\right\} .
$$

Applying 3.14 with $k, \alpha, X, f$, and $A$ replaced by $1,0, D, f \mid D$, and $B$, one obtains that $B$ is pointwise differentiable of order 1 at $a, \operatorname{Tan}(B, a) \in \mathbf{G}(n, m)$, and $S^{\perp} \cap \operatorname{Tan}(B, a)=\{0\}$, hence $\operatorname{Tan}(A, a)=\operatorname{Tan}(B, a)$. In particular, one may assume that

$$
A \subset\left\{\chi:\left|S_{\natural}^{\perp}(\chi-a)\right| \leq \kappa\left|S_{\natural}(\chi-a)\right|\right\} \quad \text { for some } 0 \leq \kappa<\infty
$$

by 3.10 . Therefore, noting 3.6. twice applying 3.11 (5) with $(A, B)$ replaced by $(A, C)$ and $(B, C)$ respectively yields the conclusion.

5.7 Theorem. Suppose $k$ and $n$ are positive integers, $m$ is an integer, $0 \leq m \leq$ $n, A \subset \mathbf{R}^{n}, 0 \leq \alpha \leq 1, \gamma=k$ if $\alpha=0$ and $\gamma=(k, \alpha)$ if $\alpha>0$, and $X$ is the set of $a \in \mathbf{R}^{n}$ such that $A$ is pointwise [strongly pointwise] differentiable of order $\gamma$ at a with $\operatorname{dim} \operatorname{Tan}(A, a)=m$.

Then the following three statements hold.

(1) There exists a countable collection of $m$ dimensional submanifolds of class $\gamma$ of $\mathbf{R}^{n}$ covering $\mathscr{H}^{m}$ almost all of $X$.

(2) If $B$ is an $m$ dimensional submanifold of class $\gamma$ of $\mathbf{R}^{n}$, then $\mathscr{H}^{m}$ almost all $a \in B \cap X$ satisfy $\mathrm{pt}^{i} A(a, \cdot)=\mathrm{ptD}^{i} B(a, \cdot)$ for $i=0, \ldots, k$ and

$$
\begin{gathered}
\lim _{r \rightarrow 0+} r^{-k-\alpha} \sup \operatorname{dist}(\cdot, B)[A \cap \mathbf{B}(a, r)]=0 . \\
{\left[\lim _{r \rightarrow 0+} r^{-k-\alpha} \sup |\operatorname{dist}(\cdot, A)-\operatorname{dist}(\cdot, B)|[\mathbf{B}(a, r)]=0 .\right]}
\end{gathered}
$$


(3) If $\alpha=1$, then $A$ is pointwise [strongly pointwise] differentiable of order $k+1$ at $\mathscr{H}^{m}$ almost all $a \in X$.

Proof. Notice that $X$ is a countably $m$ rectifiable Borel set by 5.2 and 5.5 and $X \subset$ Clos $A$ by 3.4 In view of 3.8 one may assume $m>0$.

In order to prove (1), suppose $S \in \mathbf{G}(n, m), f: S \rightarrow S^{\perp}$ is of class 1 , and define Borel sets $B=X \cap\{\chi+f(\chi): \chi \in S\}$ and $D=S \cap\{\chi: \chi+f(\chi) \in X\}$. If $a \in B, x=S_{\sharp}(a)$, and $D$ is pointwise differentiable of order 1 at $x$ with $\operatorname{Tan}(D, x)=S$, then the set $B$ is pointwise differentiable of order $\gamma$ at $a$ and

$$
\operatorname{ptD}^{i} A(a, \cdot)=\operatorname{pt} \mathrm{D}^{i} B(a, \cdot) \quad \text { for } i=0, \ldots, k
$$

by 5.6 hence 3.14 with $x, X, f$, and $A$ replaced by $S_{\natural}(a), D, f \mid D$, and $B$ yields

$$
\begin{gathered}
\lim _{r \rightarrow 0+} r^{-k} \sup \left|f-P_{x}\right|[D \cap \mathbf{B}(x, r)]=0 \quad \text { if } \alpha=0, \\
\limsup _{r \rightarrow 0+} r^{-k-\alpha} \sup \left|f-P_{x}\right|[D \cap \mathbf{B}(x, r)]<\infty \quad \text { if } \alpha>0,
\end{gathered}
$$

where $P_{x}: S \rightarrow S^{\perp}$ and $P_{x}(\chi)=\sum_{i=0}^{k}\left\langle(\chi-x)^{i} / i !\right.$, pt $\left.D^{i} A(a, S)\right\rangle$ for $\chi \in S$. Since these conditions hold for $\mathscr{H}^{m}$ almost all $a \in B$ with $x=S_{\natural}(a)$ by 3.9 there exist $g_{j}: S \rightarrow S^{\perp}$ of class $\gamma$ such that

$$
\mathscr{H}^{m}\left(D \sim \bigcup_{j=1}^{\infty}\left\{x: f(x)=g_{j}(x)\right\}\right)=0
$$

by 4.2 and 5.5 Consequently, (1) follows from [Fed69, 3.2.29].

In order to prove (2), it is sufficient to consider $B=\{\chi+f(\chi): \chi \in S\}$ corresponding to $S \in \mathbf{G}(n, m)$ and $f: S \rightarrow S^{\perp}$ of class $\gamma$ with Lip $f<\infty$ by 2.11 and [Fed69, 3.1.19(5)]. Define

$$
Y=(B \cap X) \cap\left\{y: \operatorname{ptD}^{i} A(y, \cdot)=\operatorname{pt} \mathrm{D}^{i} B(y, \cdot) \text { for } i=0, \ldots, k\right\}
$$

and $D=S \cap\{x: x+f(x) \in X\}$. In view of 3.9, twice applying 5.6 with $(A, B)$ replaced by $\left(A, B \cap S_{\natural}^{-1}[D]\right)$ and $\left(B, B \cap S_{\natural}^{-1}[D]\right)$ respectively yields

$$
\left\{x+f(x): x \in S, \Theta^{m}\left(\mathscr{H}^{m}\llcorner S \sim \operatorname{Clos} D, x)=0\right\} \subset Y, \quad \mathscr{H}^{m}(B \cap X \sim Y)=0 .\right.
$$

Let $\kappa=\operatorname{Lip} f$ and $\lambda=2^{-1}(1+\kappa)^{-1}$. For each positive integer $j$ define

$$
C_{j}=\mathbf{R}^{n} \cap\left\{a: A \cap \mathbf{U}(a, 1 / j) \subset\left\{\chi:\left|S_{\natural}^{\perp}(\chi-a)\right| \leq j\left|S_{\natural}(\chi-a)\right|\right\}\right\}
$$

and notice that $Y \subset \bigcup_{j=1}^{\infty} C_{j}$ by 3.10 Next, define for $j=1,2,3, \ldots$ sets

$$
A_{j}=A \cap\left\{\chi:\left|S_{\natural}^{\perp}(\chi)-f\left(S_{\natural}(\chi)\right)\right|<1 /(2 j)\right\}, \quad D_{j}=\left\{x: x+f(x) \in C_{j} \cap Y\right\}
$$

and functions $g_{j}: S \rightarrow\{t: 0 \leq t \leq \infty\}$ by

$$
\begin{aligned}
g_{j}(x)= & h(x), \quad\left[g_{j}(x)=\sup \left\{h(x), \operatorname{dist}\left(x+f(x), A_{j}\right)\right\},\right] \\
& \text { where } h(x)=\sup \left(\{0\} \cup\left\{\left|S_{\natural}^{\perp}(\chi)-f\left(S_{\natural}(\chi)\right)\right|: \chi \in A_{j} \cap S_{\natural}^{-1}[\{x\}]\right\}\right),
\end{aligned}
$$

for $x \in S$. The proof of $(2)$ will be concluded by showing that for each positive integer $j$ the conclusion of 2 holds at $x+f(x)$ for $\mathscr{H}^{m}$ almost all $x \in D_{j}$. 
Evidently, the set $A_{j}$ is pointwise [strongly pointwise] differentiable of order $\gamma$ at $y$ with $\operatorname{ptD}^{i} A_{j}(y, \cdot)=\operatorname{ptD}^{i} B(y, \cdot)$ for $i=0, \ldots, k$ whenever $y \in Y$ and $j$ is a positive integer. Next, it will be shown that

$$
A_{j} \cap S_{\natural}^{-1}[\mathbf{B}(x, \lambda / j)] \subset\left\{\chi:\left|S_{\natural}^{\perp}(\chi-a)\right| \leq j\left|S_{\natural}(\chi-a)\right|\right\}
$$

whenever $x \in D_{j}, a=x+f(x)$, and $j$ is a positive integer; in fact, if $\chi \in A_{j}$ and $\left|S_{\natural}(\chi)-x\right| \leq \lambda / j$, then defining $b=S_{\natural}(\chi)+f\left(S_{\natural}(\chi)\right)$ one estimates

$$
|b-a| \leq(1+\kappa)\left|S_{\natural}(b-a)\right| \leq 1 /(2 j), \quad|\chi-a| \leq|\chi-b|+|b-a|<1 / j,
$$

hence $\left|S_{\natural}^{\perp}(\chi-a)\right| \leq j\left|S_{\natural}(\chi-a)\right|$ as $a \in C_{j}$. Applying 3.11 (6) 3.11 (4) (6)] with $\kappa, A$ and $a$ replaced by $j, A_{j} \cap S_{\natural}^{-1}[\mathbf{B}(x, \lambda / j)]$ and $x+f(x)$ then yields

$$
\limsup _{r \rightarrow 0+} r^{-k-\alpha} \sup g_{j}[\mathbf{B}(x, r)]<\infty \quad \text { whenever } x \in D_{j} \text { and } j=1,2,3, \ldots
$$

implying, by 4.4 , that

$$
\lim _{r \rightarrow 0+} r^{-k-\alpha} \sup g_{j}[\mathbf{B}(x, r)]=0 \quad \text { for } \mathscr{H}^{m} \text { almost all } x \in D_{j}
$$

whenever $j$ is a positive integer. Since

$$
\begin{gathered}
\sup \operatorname{dist}(\cdot, B)\left[A_{j} \cap \mathbf{B}(a, r)\right] \leq \sup g_{j}\left[\mathbf{B}\left(S_{\natural}(a), r\right)\right] \\
{\left[\sup \left(\operatorname{dist}(\cdot, B)\left[A_{j} \cap \mathbf{B}(a, r)\right] \cup \operatorname{dist}\left(\cdot, A_{j}\right)[B \cap \mathbf{B}(a, r)]\right) \leq \sup g_{j}\left[\mathbf{B}\left(S_{\natural}(a), r\right)\right]\right]}
\end{gathered}
$$

for $a \in C_{j} \cap Y$ and $0<r<\infty$ and $A_{j}$ is a neighbourhood of $a$ relative to $A$ for such $a$, the conclusion of (2) now follows noting 3.2

To prove (3), consider $a \in B \cap X$ satisfying the conditions of (2) with respect to an $m$ dimensional submanifold $B$ of class $k+1$ of $\mathbf{R}^{n}$, as $\mathscr{H}^{m}$ almost all $a \in X$ do by (1), Fed69, 3.1.15, and (2). These conditions imply firstly that

$$
\lim _{r \rightarrow 0+} r^{-1} \sup |\operatorname{dist}(\cdot, A)-\operatorname{dist}(\cdot, B)|[\mathbf{B}(a, r)]=0
$$

by 3.10 and 3.11 (4) with $B$ replaced by $\operatorname{Tan}(A, a)$, and then that $A$ is pointwise [strongly pointwise] differentiable of order $k+1$ at $a$.

5.8 Remark. In the terminology of Anzellotti and Serapioni (1) states that $X$ is the union of a countable family whose members are " $\left(\mathscr{H}^{m}, m\right)$ rectifiable of class $\mathscr{C}^{k, \alpha} "$, see [AS94, 3.1].

\section{Approximate versus pointwise differentiation}

In this section it is shown in 6.7 that approximate differentiability of every positive integer order does not entail almost everywhere pointwise differentiability of order strictly larger than $k$ for functions of class $k$. This will be used to contrast a result on varifolds, see 7.3 and 7.4 The main lemma in this regard is 6.4 which provides for every closed set and every modulus of continuity a function of class $k$ which realises the closed set as its zero set and whose decay near that set is controlled from above and below by the given modulus of continuity.

In this section, as in [Men16a p. 993], each statement asserting the existence of a number $\Gamma$ will give rise to a function depending on the parameters determining it whose name is $\Gamma_{\mathrm{x} . \mathrm{y}}$, where x.y denotes the number of the statement. 
6.1 Lemma. Suppose $A$ is a closed subset of of $\mathbf{R}^{m}, \delta: \mathbf{R}^{m} \rightarrow \mathbf{R}$ satisfies $\delta(x)=\operatorname{dist}(x, A)$ for $x \in \mathbf{R}^{m}, U=\{x: \delta(x)<1\}$, and $k$ is a positive integer.

Then there exists a function $g: U \sim A \rightarrow \mathbf{R}$ of class $\infty$ such that

$$
\Gamma^{-1} \leq g(x) / \delta(x) \leq 1 \quad \text { and } \quad\left\|\mathrm{D}^{i} g(x)\right\| \leq \Gamma \delta(x)^{1-i} \quad \text { for } i=0, \ldots, k
$$

whenever $x \in U \sim A$, where $1 \leq \Gamma<\infty$ is determined by $k$ and $m$.

Proof. This is immediate from [Zie89, 3.6.1].

6.2 Definition. $\omega$ is termed modulus of continuity if and only if it is a function $\omega:\{t: 0 \leq t \leq 1\} \rightarrow\{t: 0 \leq t \leq 1\}$ satisfying

$$
\begin{gathered}
\lim _{t \rightarrow 0+} \omega(t)=0, \quad \omega(t)=0 \text { if and only if } t=0 \text { whenever } 0 \leq t \leq 1 \\
\omega(s) \leq \omega(t) \text { whenever } 0 \leq s \leq t \leq 1
\end{gathered}
$$

6.3 Lemma. Suppose $\omega$ is a modulus of continuity.

Then there exists a modulus of continuity $\psi$ such that $\psi \mid\{t: t>0\}$ is of class $\infty$ relative to $\{t: 0<t \leq 1\}$ and $\omega(t / 4) \leq \psi(t) \leq \omega(t)$ for $0 \leq t \leq 12$

Proof. Constructing (for instance by means of a partition of unity) a modulus of continuity $\psi$ such that $\psi \mid\{t: t>0\}$ is of class $\infty$ relative to $\{t: 0<t \leq 1\}$ and $\psi\left(2^{-i}\right)=\omega\left(2^{-i-1}\right)$ whenever $i$ is a nonnegative integer, one readily verifies the conclusion.

6.4 Lemma. Suppose $A$ is a closed subset of $\mathbf{R}^{m}, \delta: \mathbf{R}^{m} \rightarrow \mathbf{R}$ satisfies $\delta(x)=\operatorname{dist}(x, A)$ for $x \in \mathbf{R}^{m}, U=\{x: \delta(x)<1\}, \omega$ is a modulus of continuity, and $k$ is a positive integer.

Then there exists $f: U \rightarrow \mathbf{R}$ of class $k$ such that $f \mid U \sim A$ is of class $\infty$ and

$$
f(x) \geq \Gamma^{-1} \omega(\delta(x) / \Gamma) \delta(x)^{k} \quad \text { for } x \in U \sim A,
$$

$\mathrm{D}^{i} f(a)=0 \quad$ for $a \in A, \quad\left\|\mathrm{D}^{i} f(x)\right\| \leq \Gamma \omega(\delta(x)) \delta(x)^{k-i} \quad$ for $x \in U \sim A$

whenever $i=0, \ldots, k$, where $1 \leq \Gamma<\infty$ is determined by $k$ and $m$.

Proof. In view of 6.3 the problem reduces to the case that $\omega \mid\{t: t>0\}$ is of class $\infty$ relative to $\{t: 0<t \leq 1\}$.

In this case there exists a function $h:\{y: y<1\} \rightarrow \mathbf{R}$ of class $k$ such that ${ }^{23}$

$$
h(y)=0 \quad \text { for }-\infty<y \leq 0, \quad h^{(k)}(y)=\omega(y) \quad \text { for } 0 \leq y<1 .
$$

Then [Fed69, 3.1.11 implies

$$
h^{(i)}(y)=\left(y^{k-i} /(k-i) !\right) \int_{0}^{1}(k-i)(1-t)^{k-i-1} \omega(t y) \mathrm{d} \mathscr{L}^{1} t
$$

for $0 \leq y<1$ and $i=0, \ldots, k$. Consequently, one obtains the estimates

$$
\begin{gathered}
h(y) \geq\left(y^{k} / k !\right) \omega(y / 2) \int_{1 / 2}^{1} k(1-t)^{k-1} \mathrm{~d} \mathscr{L}^{1} t=2^{-k} \omega(y / 2) y^{k} / k !, \\
\left|h^{(i)}(y)\right| \leq \omega(y) y^{k-i} /(k-i) ! \quad \text { for } i=0, \ldots, k
\end{gathered}
$$

\footnotetext{
${ }^{22}$ If $A \subset \mathbf{R}$ and $f: A \rightarrow \mathbf{R}$ then $f$ is of class $\infty$ relative to $A$ if and only if there exist an open subset $U$ of $\mathbf{R}$ and $g: U \rightarrow \mathbf{R}$ of class $\infty$ with $A \subset U$ and $f=g \mid A$, see [Fed69, 3.1.22].

${ }^{23}$ If $g$ maps a subset of $\mathbf{R}$ into $\mathbf{R}$ and $g$ is $k$ times differentiable at $x$, then $g^{(k)}(x) \in \mathbf{R}$ denotes the $k$-th derivative of $g$ at $x$, see Fed69 3.1.11.
} 
whenever $0 \leq y<1$.

Next, choose $g$ as in 6.1 abbreviate $\Delta=\Gamma_{[6.1]}(k, m)$, and define $f: U \rightarrow \mathbf{R}$ by $f(a)=0$ for $a \in A$ and $f(x)=h(g(x))$ for $x \in U \sim A$. Defining $S(i)$ to be the set of all $k$ termed sequences $\alpha$ of nonnegative integers with $\sum_{j=1}^{k} j \alpha_{j}=i$, one estimates, using [Fed69, 1.10.5 3.1.11, the estimates for $h$, and 6.1.

$$
\begin{aligned}
\left\|\mathrm{D}^{i} f(x)\right\| / i ! & \leq \sum_{\alpha \in S(i)}\left|h^{\left(\sum \alpha\right)}(g(x))\right| \prod_{j=1}^{k}\left(\left\|\mathrm{D}^{j} g(x)\right\| / j !\right)^{\alpha_{j}} /\left(\alpha_{j}\right) ! \\
& \leq \omega(g(x)) \sum_{\alpha \in S(i)}\left(g(x)^{k-\sum \alpha} /\left(k-\sum \alpha\right) !\right)\left(\Delta \sum \alpha / \alpha !\right) \delta(x)^{\left(\sum \alpha\right)-i} \\
& \leq \Delta_{i} \omega(\delta(x)) \delta(x)^{k-i},
\end{aligned}
$$

for $x \in U \sim A$ and $i=0, \ldots, k$, where $\Delta_{i}=\sum_{\alpha \in S(i)}\left(\Delta \sum \alpha / \alpha !\right) /\left(k-\sum \alpha\right) !$. Inductively one infers that $f$ is $i$ times differentiable with $\mathrm{D}^{i} f(a)=0$ for $a \in A$ and $\mathrm{D}^{i} f$ is continuous for $i=0, \ldots, k$. Since the estimates for $h$ and 6.1 yield

$f(x) \geq 2^{-k} \omega(g(x) / 2) g(x)^{k} / k ! \geq(2 \Delta)^{-k} \omega(\delta(x) /(2 \Delta)) \delta(x)^{k} / k ! \quad$ for $x \in U \sim A$,

one may take $\Gamma=\sup \left(\left\{(2 \Delta)^{k} k !\right\} \cup\left\{\Delta_{i} i !: i=0, \ldots, k\right\}\right)$ in the present case.

6.5 (see for instance [KM17, 7.8]). If $m$ is a positive integer, then $\boldsymbol { \alpha } ( m ) \leq 6 \longdiv { { } ^ { 2 4 } }$

6.6 (see [Fed69, 5.1.9]). Suppose $m$ is a positive integer and $n=m+1$. Then $\mathbf{p}: \mathbf{R}^{n} \rightarrow \mathbf{R}^{m}$ and $\mathbf{q}: \mathbf{R}^{n} \rightarrow \mathbf{R}$ satisfy $\mathbf{p}(z)=\left(z_{1}, \ldots, z_{m}\right)$ and $\mathbf{q}(z)=z_{n}$ for $z=\left(z_{1}, \ldots, z_{n}\right) \in \mathbf{R}^{n}$.

6.7 Example. Suppose $k$ and $m$ are positive integers.

Then there exist a closed subset $A$ of $\mathbf{R}^{m}$ and a nonnegative function $f$ : $\mathbf{R}^{m} \rightarrow \mathbf{R}$ of class $k$ satisfying

$$
\begin{gathered}
\mathrm{D}^{i} f(a)=0 \quad \text { whenever } a \in A \text { and } i=0, \ldots, k, \quad \mathscr{L}^{m}(A)>0, \\
\limsup _{x \rightarrow a}|x-a|^{-k-\alpha} f(x)=\infty \text { for } 0<\alpha \leq 1 \quad \text { for } \mathscr{L}^{m} \text { almost all } a \in A .
\end{gathered}
$$

In particular, if $0<\alpha \leq 1$, then $\mathbf{R}^{n} \cap\{z: \mathbf{q}(z)=f(\mathbf{p}(z))\}$ is not pointwise differentiable of order $(k, \alpha)$ at $c$ for $\mathscr{H}^{m}$ almost all $c \in \mathbf{p}^{*}[A]$, see $6.6{ }^{25}$

Construction. Evidently, it is sufficient to prove the assertion with " $f: \mathbf{R}^{m} \rightarrow \mathbf{R}$ " replaced by " $f:\{x: \operatorname{dist}(x, A)<1\} \rightarrow \mathbf{R}$ ". Define a modulus of continuity $\omega$ by

$$
\omega(0)=0, \quad \omega(t)=(1+\log (1 / t))^{-1} \quad \text { for } 0<t \leq 1 .
$$

By Kolasiński and the author [KM17, 2.5], there exist $B \subset\{r: r>0\}$, a Borel subset $X$ of $\mathbf{R}^{m}$, and a collection $F$ of open balls in $\mathbf{R}^{m}$ such that

$$
\inf B=0, \quad \mathscr{L}^{m}(X)>0, \quad \mathscr{L}^{m}((\operatorname{Clos} X) \sim X)=0,
$$

\footnotetext{
${ }^{24}$ By definition $\boldsymbol{\alpha}(m)=\mathscr{L}^{m} \mathbf{B}(0,1)$, see [Fed69, 2.7.16 (1)].

${ }^{25}$ The adjoint linear map $\mathbf{p}^{*}: \mathbf{R}^{m} \rightarrow \mathbf{R}^{n}$ associated to $\mathbf{p}$ satisfies $\mathbf{p}^{*}(x)=\left(x_{1}, \ldots, x_{m}, 0\right) \in$ $\mathbf{R}^{n}$ for $x=\left(x_{1}, \ldots, x_{m}\right) \in \mathbf{R}^{m}$, see [Fed69, 1.7.4].
} 
and such that for $a \in X$ and $r \in B$ there exists $U \in F$ with

$$
U \subset \mathbf{U}(a, r) \sim X \quad \text { and } \quad \mathscr{L}^{m}(U) \geq \omega(r) r^{m} .
$$

Define $A=\operatorname{Clos} X$, take $\delta$ and $f$ as in 6.4 and let $\Delta=6^{k} \Gamma_{[6.4}(k, m)$. If $a \in X$, $r \in B$, and $U=\mathbf{U}(x, s) \in F$ are related as above then one estimates, using 6.5

$$
\begin{gathered}
|x-a| \leq r, \quad \delta(x) \geq s \geq \omega(r)^{1 / m} \boldsymbol{\alpha}(m)^{-1 / m} r \geq \omega(|x-a|)^{1 / m}|x-a| / 6, \\
f(x) \geq \Delta^{-1} \omega\left(\omega(|x-a|)^{1 / m}|x-a| / \Delta\right) \omega(|x-a|)^{k / m}|x-a|^{k} .
\end{gathered}
$$

The principal conclusion now follows from the definition of $\omega$ and the postscript is a consequence of 3.14 .

\section{Intersecting a stationary varifold with a plane}

In this section a regularity property of the support of the weight measure of a stationary integral varifold is proven in 7.2 Namely, if near one of its points it is contained in the union of a plane of the same dimension as the varifold and a set with density zero at that point then it is strongly pointwise differentiable of every positive integer order at that point. This applies to almost all points of the intersection with such a plane, see 7.3 The differentiability condition obtained not only encodes a vanishing phenomenon of infinite order but additionally places a strong restriction on the size of "holes" the varifold may have near that point both with respect to distance and Hausdorff measure, see 3.10, 3.11, 6), and Kolasiński and the author [KM17, 10.4, 11.7, 11.8].

7.1 Lemma. Suppose $m$ and $n$ are positive integers, $2 \leq m \leq n, a \in \mathbf{R}^{n}$, $0<r<\infty, V \in \mathbf{V}_{m}(\mathbf{U}(a, r)), \delta V=0, \Theta^{m}(\|V\|, x) \geq 1$ for $\|V\|$ almost all $x$, $T \in \mathbf{G}(n, m), 0<l<\infty$,

$$
\|V\|(\mathbf{U}(a, s) \sim\{x: x-a \in T\}) \leq 2^{-m-l} \boldsymbol{\alpha}(m) s^{m} \quad \text { for } 0<s \leq r,
$$

and $\psi(s)=\sup \{\operatorname{dist}(x-a, T): x \in \mathbf{U}(a, s) \cap \operatorname{spt}\|V\|\}$ for $0<s \leq r$.

Then there holds

$$
\psi(s) \leq 2^{l}(s / r)^{l} \psi(r) \quad \text { for } 0<s \leq r .
$$

Proof. If $f: \mathbf{U}(a, r) \rightarrow \mathbf{R}$ is a convex function, then

$$
\int f(x) D(\operatorname{grad} \zeta)(x) \bullet S_{\natural} \mathrm{d} V(x, S) \geq 0 \quad \text { for } 0 \leq \zeta \in \mathscr{D}(\mathbf{U}(a, r), \mathbf{R}) ;
$$

in fact, convolution reduces the problem to the case that $f$ is of class $\infty$ which follows from Allard [All72, 7.5(1) (2)]. Take $f(x)=\operatorname{dist}(x-a, T)$ for $x \in \mathbf{U}(a, r)$, abbreviating $\phi(s)=s^{-m} \int_{\mathbf{U}(a, s)} f \mathrm{~d}\|V\|$ for $0<s \leq r$, and denote by $e_{1}, \ldots, e_{n}$ the standard orthonormal base of $\mathbf{R}^{n}$. Applying Michael and Simon [MS73, 3.4] with $M, U, \mu, \tilde{g}^{i j}(x), \mathscr{H}_{i}(x), \chi, \Lambda, \xi$, and $\varrho$ replaced by $\mathbf{U}(a, r), \mathbf{U}(a, r),\|V\|$, $\operatorname{Tan}^{m}(\|V\|, x)_{\natural}\left(e_{i}\right) \bullet e_{j}, 0, f, 0, x$, and $s / 2$ for $x \in \mathbf{U}(a, s / 2) \cap$ spt $\|V\|$ implies

$$
\psi(s / 2) \leq \boldsymbol{\alpha}(m)^{-1} 2^{m} \phi(s) \quad \text { for } 0<s \leq r .
$$

Moreover, Hölder's inequality yields

$$
\phi(s) \leq 2^{-m-l} \boldsymbol{\alpha}(m) \psi(s) \text { for } 0<s \leq r .
$$


Together one obtains the conclusion; in fact, it is evident if $s \geq r / 2$ and if it holds for some $0<s \leq r$ then

$$
\psi(s / 2) \leq 2^{-l} \psi(s) \leq(s / r)^{l} \psi(r)
$$

and the conclusion holds for $s / 2$.

7.2 Theorem. Suppose $m$ and $n$ are positive integers, $2 \leq m \leq n, U$ is an open subset of $\mathbf{R}^{n}, V \in \mathbf{I V}_{m}(U), \delta V=0$, and

$$
a \in \operatorname{spt}\|V\|, \quad T \in \mathbf{G}(n, m), \quad \boldsymbol{\Theta}^{m}(\|V\|\llcorner U \cap\{x: x-a \notin T\}, a)=0 .
$$

Then spt $\|V\|$ is strongly pointwise differentiable of every positive integer order at $a, \operatorname{Tan}(\operatorname{spt}\|V\|, a)=T$, and

$$
\lim _{s \rightarrow 0+} s^{-l} \int_{\mathbf{U}(a, s) \times \mathbf{G}(n, m)}\left\|S_{\natural}-T_{\natural}\right\|^{2} \mathrm{~d} V(x, S)=0 \quad \text { for } 0<l<\infty .
$$

Proof. Assume $a=0$ and abbreviate $A=\operatorname{spt}\|V\|$. Then 7.1 yields

$$
\lim _{s \rightarrow 0+} s^{-l} \sup \operatorname{dist}(\cdot, T)[A \cap \mathbf{B}(0, s)]=0 \text { for } 0<l<\infty,
$$

in particular one may assume that $A \subset\left\{\chi:\left|T_{\natural}^{\perp}(\chi)\right| \leq\left|T_{\natural}(\chi)\right|\right\}$. Notice that $1 \leq \boldsymbol{\Theta}^{m}(\|V\|, 0)<\infty$ by Allard [All72, 5.1 (2), 8.6]. One infers

$$
\lim _{s \rightarrow 0+} s^{-m-2(l-1)} \int_{\mathbf{U}(0, s) \times \mathbf{G}(n, m)}\left\|S_{\natural}-T_{\natural}\right\|^{2} \mathrm{~d} V(x, S)=0 \quad \text { for } 0<l<\infty
$$

from Allard All72, 8.13]. This implies

$$
\lim _{s \rightarrow 0+} s^{-m} \int f\left(s^{-1} x, S\right) \mathrm{d} V(x, S)=\mathbf{\Theta}^{m}(\|V\|, 0) \int_{T} f(x, T) \mathrm{d} \mathscr{H}^{m} x
$$

whenever $f: \mathbf{R}^{n} \times \mathbf{G}(n, m) \rightarrow \mathbf{R}$ is a continuous function with compact support and that $\boldsymbol{\Theta}^{m}(\|V\|, 0)$ is an integer by Allard [All72, 3.4, 4.6 (3), 6.4], hence one observes that

$$
\begin{gathered}
\lim _{s \rightarrow 0+} s^{-m-2(l-1)} \mathscr{H}^{m}\left(T \cap \mathbf{B}(0, s) \sim T_{\mathrm{\natural}}[A]\right)=0, \\
\lim _{s \rightarrow 0+} s^{-1-2(l-1) / m} \sup \operatorname{dist}\left(\cdot, T_{\mathrm{\natural}}[A]\right)[T \cap \mathbf{B}(0, s)]=0
\end{gathered}
$$

for $0<l<\infty$ by Kolasiński and the author KM17, 10.4]. Therefore $T_{\mathrm{a}}[A]$ is strongly pointwise differentiable of every positive integer order at 0 and $\operatorname{Tan}\left(T_{\mathrm{\natural}}[A], 0\right)=T$ by 3.2 and 3.4 Now, the conclusion follows from 3.11(5) with $S$ and $B$ replaced by $T$ and $T$.

7.3 Corollary. Suppose $m$ and $n$ are positive integers, $2 \leq m \leq n$, $U$ is an open subset of $\mathbf{R}^{n}, V \in \mathbf{I V}_{m}(U), \delta V=0$, and $T \in \mathbf{G}(n, m)$.

Then spt $\|V\|$ is strongly pointwise differentiable of every positive integer order at $a, \operatorname{Tan}(\operatorname{spt}\|V\|, a)=T$, and

$$
\lim _{s \rightarrow 0+} s^{-l} \int_{\mathbf{U}(a, s) \times \mathbf{G}(n, m)}\left\|S_{\natural}-T_{\natural}\right\|^{2} \mathrm{~d} V(x, S)=0 \quad \text { for } 0<l<\infty
$$

for $\mathscr{H}^{m}$ almost all $a \in T \cap U$.

Proof. This is a consequence of [Fed69, 2.10.19(4)] and 7.2

7.4 Remark. The behaviour of spt $\|V\|$ exhibited in 7.3 is not shared by all closed $m$ dimensional submanifolds of $\mathbf{R}^{m+1}$ of class 2 by 6.7 with $k=2$.

7.5 Remark. In case $m=1$ a complete description of the structure $\mathscr{H}^{1}$ almost everywhere of spt $\|V\|$ was obtained by Allard and Almgren in [AA76, p. 89]. 


\section{A Items employed from Federer's treatise}

For the convenience of the reader, Table 1 provides a brief list of the results employed from [Fed69. Items which merely provide background are not listed.

\begin{tabular}{|ll|}
\hline Number & Description \\
\hline 1.10 .2 & Algebra of symmetric forms. \\
1.10 .4 & Polynomial functions and Taylor's formula. \\
2.2 .7 & Estimates of seminorms related to symmetric forms. \\
2.2 .10 & Masic properties of Lipschitzian maps. \\
2.7 .1 & Transitive left actions on homogeneous spaces. \\
2.9 .17 & A differentiation theorem for general measures. \\
2.10 .19 & Properties of densities. \\
2.10 .21 & Includes Ascoli theorem for Lipschitzian functions. \\
3.1 .1 & First order differentials. \\
3.1 .11 & Higher differentials: Taylor formula, $k$ jets, composition formula. \\
3.1 .14 & Whitney's extension theorem. \\
3.1 .15 & Lusin type approximation of functions of class $(k, 1)$ by functions \\
& of class $k+1$, see also Whitney [Whi51, Theorem 4 ]. \\
3.1 .18 & Consequences of the inverse function theorem. \\
3.1 .19 & Characterisations of submanifolds of Euclidean space. \\
3.1 .21 & Tangent cones and their mapping properties. \\
3.2 .28 & Includes Grassmann manifolds treated as homogeneous spaces. \\
3.2 .29 & Characterising countably ( $\mathscr{H} m, m)$ rectifiable sets by coverings \\
3.3 .5 & consisting of submanifolds of class 1. \\
\hline
\end{tabular}

Table 1: Items employed from [Fed69].

\section{References}

[AA76] W. K. Allard and F. J. Almgren, Jr. The structure of stationary one dimensional varifolds with positive density. Invent. Math., 34(2):8397, 1976. URL: https://doi.org/10.1007/BF01425476

[AF09] Jean-Pierre Aubin and Hélène Frankowska. Set-valued analysis. Modern Birkhäuser Classics. Birkhäuser Boston, Inc., Boston, MA, 2009. Reprint of the 1990 edition [MR1048347]. URL: https: //doi.org/10.1007/978-0-8176-4848-0.

[All72] William K. Allard. On the first variation of a varifold. Ann. of Math. (2), 95:417-491, 1972. URL: https://doi.org/10.2307/1970868

[Alm00] Frederick J. Almgren, Jr. Almgren's big regularity paper, volume 1 of World Scientific Monograph Series in Mathematics. World Scientific Publishing Co. Inc., River Edge, NJ, 2000. $Q$-valued functions minimizing Dirichlet's integral and the regularity of area-minimizing rectifiable currents up to codimension 2 , With a preface by Jean 
E. Taylor and Vladimir Scheffer. URL: https://doi.org/10.1142/ 9789812813299 .

[AS94] Gabriele Anzellotti and Raul Serapioni. $\mathcal{C}^{k}$-rectifiable sets. J. Reine Angew. Math., 453:1-20, 1994. URL: https://doi.org/10.1515/ crll.1994.453.1.

[Bee85] Gerald Beer. On convergence of closed sets in a metric space and distance functions. Bull. Austral. Math. Soc., 31(3):421-432, 1985. URL: https://doi.org/10.1017/S0004972700009370.

[Bra78] Kenneth A. Brakke. The motion of a surface by its mean curvature, volume 20 of Mathematical Notes. Princeton University Press, Princeton, N.J., 1978. URL: http://www.jstor.org/stable/ j.ctt130hk4d

[Cam64] S. Campanato. Proprietà di una famiglia di spazi funzionali. Ann. Scuola Norm. Sup. Pisa (3), 18:137-160, 1964. URL: http://www. numdam.org/item?id=ASNSP_1964_3_18_1_137_0

[CCKŚ96] L. Caffarelli, M. G. Crandall, M. Kocan, and A. Świẹch. On viscosity solutions of fully nonlinear equations with measurable ingredients. Comm. Pure Appl. Math., 49(4):365-397, 1996.

URL: https ://doi.org/10.1002/(SICI) 1097-0312(199604) 49:4 $<365:$ :AID-CPA3>3.3.CO; $2-\mathrm{V}$.

[CZ61] A.-P. Calderón and A. Zygmund. Local properties of solutions of elliptic partial differential equations. Studia Math., 20:171-225, 1961. URL: http://matwbn.icm.edu.pl/ksiazki/sm/sm20/sm20113.pdf

[Dav03] Guy David. Limits of Almgren quasiminimal sets. In Harmonic analysis at Mount Holyoke (South Hadley, MA, 2001), volume 320 of Contemp. Math., pages 119-145. Amer. Math. Soc., Providence, RI, 2003. URL: https://doi.org/10.1090/conm/320/05603

[DS58] Nelson Dunford and Jacob T. Schwartz. Linear Operators. I. General Theory. With the assistance of W. G. Bade and R. G. Bartle. Pure and Applied Mathematics, Vol. 7. Interscience Publishers, Inc., New York; Interscience Publishers, Ltd., London, 1958. URL: https://babel.hathitrust.org/cgi/pt?id=mdp. 39015000962400; view=1up; seq=9A.

[EG15] Lawrence C. Evans and Ronald F. Gariepy. Measure theory and fine properties of functions. Textbooks in Mathematics. CRC Press, Boca Raton, FL, revised edition, 2015.

[Fed69] Herbert Federer. Geometric measure theory. Die Grundlehren der mathematischen Wissenschaften, Band 153. Springer-Verlag New York Inc., New York, 1969. URL: https://doi.org/10.1007/ 978-3-642-62010-2

[HS75] Edwin Hewitt and Karl Stromberg. Real and abstract analysis. Springer-Verlag, New York-Heidelberg, 1975. A modern treatment of the theory of functions of a real variable, Third printing, Graduate 
Texts in Mathematics, No. 25. URL: https://doi.org/10.1007/ 978-3-642-88044-5.

[Ilm96] T. Ilmanen. A strong maximum principle for singular minimal hypersurfaces. Calc. Var. Partial Differential Equations, 4(5):443-467, 1996. URL: https://doi.org/10.1007/s005260050049

[Isa87a] N. M. Isakov. On a global property of approximately differentiable functions. Mathematical Notes, 41(4):280-285, 1987. URL: https: //doi.org/10.1007/BF01137673.

[Isa87b] N. M. Isakov. On a global property of approximately differentiable functions. (Russian). Mat. Zametki, 41(4):500-508, 1987. URL: http://mi.mathnet.ru/eng/mz4871

[Jár85] A. Járai. Derivatives are Borel functions. Aequationes Math., 29(1):2427, 1985. URL: https://doi.org/10.1007/BF02189809.

[Kec95] Alexander S. Kechris. Classical descriptive set theory, volume 156 of Graduate Texts in Mathematics. Springer-Verlag, New York, 1995. URL: https://doi.org/10.1007/978-1-4612-4190-4.

[Kel75] John L. Kelley. General topology. Springer-Verlag, New York, 1975. Reprint of the 1955 edition [Van Nostrand, Toronto, Ont.], Graduate Texts in Mathematics, No. 27.

[KM17] Sławomir Kolasiński and Ulrich Menne. Decay rates for the quadratic and super-quadratic tilt-excess of integral varifolds. NoDEA Nonlinear Differential Equations Appl., 24(2):Art. 17, 56, 2017. URL: https: //doi.org/10.1007/s00030-017-0436-z.

[Liu08] Fon-Che Liu. A localized Lusin theorem and a Rademacher type theorem. Bull. Inst. Math. Acad. Sin. (N.S.), 3(2):243-253, 2008. URL: http://w3.math.sinica.edu.tw/bulletin_ns/20082/ 2008201.pdf

[LL13] Chun-Liang Lin and Fon-Che Liu. Approximate differentiability according to Stepanoff-Whitney-Federer. Indiana Univ. Math. J., 62(3):855-868, 2013. URL: https://doi.org/10.1512/iumj.2013 62.5024

[Men09] Ulrich Menne. Some applications of the isoperimetric inequality for integral varifolds. Adv. Calc. Var., 2(3):247-269, 2009. URL: https://doi.org/10.1515/ACV.2009.010

[Men10] Ulrich Menne. A Sobolev Poincaré type inequality for integral varifolds. Calc. Var. Partial Differential Equations, 38(3-4):369-408, 2010. URL: https://doi.org/10.1007/s00526-009-0291-9.

[Men12a] Ulrich Menne. Decay estimates for the quadratic tilt-excess of integral varifolds. Arch. Ration. Mech. Anal., 204(1):1-83, 2012. URL: https: //doi.org/10.1007/s00205-011-0468-1. 
[Men12b] Ulrich Menne. A sharp lower bound on the mean curvature integral with critical power for integral varifolds, 2012. In abstracts from the workshop held July 22-28, 2012, Organized by Camillo De Lellis, Gerhard Huisken and Robert Jerrard, Oberwolfach Reports. Vol. 9, no. 3. URL: https://doi.org/10.4171/OWR/2012/36

[Men13] Ulrich Menne. Second order rectifiability of integral varifolds of locally bounded first variation. J. Geom. Anal., 23(2):709-763, 2013. URL: https://doi.org/10.1007/s12220-011-9261-5.

[Men16a] Ulrich Menne. Weakly differentiable functions on varifolds. Indiana Univ. Math. J., 65(3):977-1088, 2016. URL: https://doi.org/10. 1512/iumj.2016.65.5829

[Men16b] Ulrich Menne. Sobolev functions on varifolds. Proc. Lond. Math. Soc. (3), 113(6):725-774, 2016. URL: https://doi.org/10.1112/plms/ pdw023.

[Men18] Ulrich Menne. Pointwise differentiability of higher order for distributions, 2018. arXiv:arXiv:1803.10855v1

[MS73] J. H. Michael and L. M. Simon. Sobolev and mean-value inequalities on generalized submanifolds of $R^{n}$. Comm. Pure Appl. Math., 26:361379, 1973. URL: https://doi.org/10.1002/cpa.3160260305

[O'N63] Richard O'Neil. Convolution operators and $L(p, q)$ spaces. Duke Math. J., 30:129-142, 1963. URL: http://projecteuclid.org/euclid. $\mathrm{dmj} / 1077374532$

[Reš68a] Ju. G. Rešetnjak. Generalized derivatives and differentiability almost everywhere. Math. USSR-Sb., 4:293-302, 1968. URL: https://doi. org/10.1070/SM1968v004n03ABEH002799.

[Reš68b] Yu. G. Reshetnyak. Generalized derivatives and differentiability almost everywhere. (Russian). Mat. Sb. (N.S.), 75(117):323-334, 1968. URL: http://mi.mathnet.ru/eng/msb3987.

[San17] Mario Santilli. Rectifiability and approximate differentiability of higher order for sets. Indiana Univ. Math. J., 2017. To appear, available at https://arxiv.org/abs/1701.07286v2.

[Sch04] Reiner Schätzle. Quadratic tilt-excess decay and strong maximum principle for varifolds. Ann. Sc. Norm. Super. Pisa Cl. Sci. (5), 3(1):171-231, 2004. URL: http://www.numdam.org/item?id= ASNSP_2004_5_3_1_171_0

[Sch09] Reiner Schätzle. Lower semicontinuity of the Willmore functional for currents. J. Differential Geom., 81(2):437-456, 2009. URL: http: //projecteuclid.org/getRecord?id=euclid.jdg/1231856266.

[Ste70] Elias M. Stein. Singular integrals and differentiability properties of functions. Princeton Mathematical Series, No. 30. Princeton University Press, Princeton, N.J., 1970. URL: https://www.degruyter. com/view/product/481471 
[Ste81] E. M. Stein. Editor's note: the differentiability of functions in $\mathbf{R}^{n}$. Ann. of Math. (2), 113(2):383-385, 1981.

URL: http://links . jstor.org/sici?sici=0003-486X (198103) 2 : 113:2\%3C383:ENTDOF\%3E2.0.CD;2-4\&origin=MSN.

[SW89] Bruce Solomon and Brian White. A strong maximum principle for varifolds that are stationary with respect to even parametric elliptic functionals. Indiana Univ. Math. J., 38(3):683-691, 1989. URL: https://doi.org/10.1512/iumj.1989.38.38032.

[Tru89] Neil S. Trudinger. On the twice differentiability of viscosity solutions of nonlinear elliptic equations. Bull. Austral. Math. Soc., 39(3):443447, 1989. URL: https://doi.org/10.1017/S0004972700003361.

[Wei95] Clifford E. Weil. The Peano notion of higher order differentiation. Math. Japon., 42(3):587-600, 1995.

[Whi51] Hassler Whitney. On totally differentiable and smooth functions. Pacific J. Math., 1:143-159, 1951. URL: https://projecteuclid org/euclid.pjm/1102613161

[Whi10] Brian White. The maximum principle for minimal varieties of arbitrary codimension. Comm. Anal. Geom., 18(3):421-432, 2010. URL: https://doi.org/10.4310/CAG.2010.v18.n3.a1.

[Wic14a] Neshan Wickramasekera. A general regularity theory for stable codimension 1 integral varifolds. Ann. of Math. (2), 179(3):843-1007, 2014. URL: https://doi.org/10.4007/annals.2014.179.3.2

[Wic14b] Neshan Wickramasekera. A sharp strong maximum principle and a sharp unique continuation theorem for singular minimal hypersurfaces. Calc. Var. Partial Differential Equations, 51(3-4):799-812, 2014. URL: https://doi.org/10.1007/s00526-013-0695-4.

[Zib78] Israel Bernard Zibman. Some characterizations of the $n$-dimensional Peano derivative. Studia Math., 63(1):89-110, 1978. URL: https: //doi.org/10.4064/sm-63-1-89-110

[Zie89] William P. Ziemer. Weakly differentiable functions, volume 120 of Graduate Texts in Mathematics. Springer-Verlag, New York, 1989. Sobolev spaces and functions of bounded variation. URL: https: //doi.org/10.1007/978-1-4612-1015-3.

\section{AfFiliations}

Institute of Mathematics, University of Leipzig

Augustusplatz 10, 04109 LEIPZIG, GERMANY

Max Planck Institute for Mathematics in the Sciences Inselstraße 22, 04103 LeIPZIG, Germany

EMAIL ADDRESSES

Ulrich.Menne@math.uni-leipzig.de Ulrich.Menne@mis.mpg.de 